\title{
Finiteness of Crystalline Cohomology of Higher Level
}

\author{
Kazuaki MIYATANI
}

\begin{abstract}
We prove the finiteness of crystalline cohomology of higher level. An important ingredient is a "higher de Rham complex" and a kind of Poincaré lemma for it.
\end{abstract}

\section{Introduction.}

\subsection{Crystalline cohomology of higher level.}

Pierre Berthelot [B3] generalized the notion of PD structure, the most fundamental notion in the crystalline theory, to that of " $m$-PD structure (PD structure of level $m$ )" for each natural number $m$. After replacing the classical PD structure by this notion, we directly get a level- $m$ version of crystalline site, crystal and other crystalline concepts. In particular, we can define $m$-crystalline cohomology over very ramified DVR because a DVR of mixed characteristic $(0, p)$ has an $m$-PD structure on its maximal ideal if the absolute ramification index is not greater than $p^{m}(p-1)$.

In contrast to the simplicity of this generalization, we cannot directly apply classical arguments to prove fundamental properties of the $m$-crystalline cohomology such as base change and finiteness. The main reason lies in the fact that the crystalline Poincaré lemma appears to be difficult in the level- $m$ situation. Bernard Le Stum and Adolfo Quirós indeed proved the so-called exact crystalline Poicaré lemma [LS-Q2], which states that the $m$-crystalline cohomology is calculated by the "jet complex of order $p^{m}$ "; unfortunately, this complex is not bounded and its local freeness is yet to be proved (the latter point will be discussed in the next subsection). The lack of boundedness and local freeness prevents us from proving as in the classical case the cohomological boundedness and the base change, which we need for proving, for example, the finiteness.

In spite of this difficulty, we prove in this article the finiteness of the cohomology by using an auxiliary "de Rham-like" complex and corresponding Poincaré lemma. To be more precise, we first define in a local situation a complex that is, if we ignore the differential maps, isomorphic to the usual de Rham complex; we call this complex the "higher de Rham complex." We next prove that this is a resolution of a direct sum of finitely many copies of the structure sheaf. Thirdly, although the arguments so far are done just locally, we show in the global situation the cohomological boundedness and the base change theorem. At last, by using these two properties 
and the exact crystalline Poincaré lemma, we prove the finiteness of this cohomology.

This article is organized as follows. Section 1 is devoted to recalling the foundation of $m$-crystalline theory and doing some differential calculi. In Section 2 introduced is the higher de Rham complex and the Poincaré lemma for it, and in Section 3 proved is the finiteness of $m$-crystalline cohomology. Finally, other applications of the higher de Rham complex are included in Section 4.

\subsection{A problem on local freeness of the jet complex.}

In fact, if $(S, \mathfrak{a}, \mathfrak{b}, \gamma)$ is an $m$-PD scheme (Definition 1.1) and if $X$ is a smooth scheme over S, Le Stum and Quirós [LS-Q2, 1.4] give a proof of the local freeness of each term appearing in the jet complex of order $p^{m}$, whose $r$-th term is there denoted by $\Omega_{X_{m}}^{r}$. However, the proof for $r \geq 3$ is not correct.

Indeed, let $n$ be the dimension of $X$ over $S$, and $t_{1}, \ldots, t_{n}$ a system of local coordinates. Then, as proved in the article, there exist, for each $I \in \mathbb{N}^{n}$ that satisfies $p^{m}<|I| \leq 2 p^{m}$, two multi-indices $A(I), B(I)$ such that $A(I)+B(I)=I$ and that

$$
\left\{(d t)^{U} \otimes(d t)^{V} \mid(U, V) \neq(A(U+V), B(U+V))\right\}
$$

is a basis of $\Omega_{X_{m}}^{2}$. By using this notation, the module $\Omega_{X_{m}}^{3}$ is generated by the set

$$
\left\{(d t)^{U} \otimes(d t)^{V} \otimes(d t)^{W} \mid(V, W) \neq(A(V+W), B(V+W))\right. \text { and, }
$$

if $W$ is not $B(I)$ for any $I$, then $(U, V) \neq(A(U+V), B(U+V))\}$.

Their relations are given by, for each $(U, V, W)$ such that $(U, V)=(A(U+V), B(U+V))$ and that $W=B(I)$ for some $I$,

$$
\begin{aligned}
\sum_{S}\left\langle\begin{array}{c}
U+V \\
S
\end{array}\right\rangle(d t)^{U+V-S} \otimes(d t)^{S} \otimes(d t)^{W} \\
\quad-\sum_{I}\left\langle\begin{array}{c}
U+V \\
A(I)
\end{array}\right\rangle\left\langle\begin{array}{c}
I \\
A(I)
\end{array}\right\rangle^{-1} \sum_{\substack{0<T<I \\
T \neq A(I)}}\left\langle\begin{array}{l}
I \\
T
\end{array}\right\rangle(d t)^{U+V-A(I)} \otimes(d t)^{T} \otimes(d t)^{I-T}=0,
\end{aligned}
$$

where the first sum is taken over all $S$ such that $0<S<U+V$ and that $S$ is not equal to $A(I)$ for any $I$ with $W=B(I)$, and where the first sum in the second line moves $I$ such that $W=B(I)$; three relatons written in that article are not correct.

Then, we have two problems with these relations. First, it may happen that all the coefficients in this sum are non-unit. Second, even if one of the coefficients in the first sum is a unit (this assumption holds, for example, if there exists only one $I$ that satisfies $W=B(I)), I-T$ in the second sum may again be of the form $B\left(I^{\prime}\right)$; at this point, we do not know how to exclude the auxiliary generators by these relations to prove the local freeness.

We refer to influences of this problem on other results in their article [LS-Q2].

First, it does not affect the exact Poincaré lemma at the level of $\mathscr{O}_{X}$-modules [LS-Q2, 3.3], whose proof does not use the local freeness. Second, although the proof of the exact Poincaré lemma with coefficients [LS-Q2, 4.7] fails in general, it remains 
valid if the coefficient $E$ is a flat $m$-crystal since then the assertion is reduced to the case where $E=\mathscr{O}_{X / S}^{(m)}$. Finally, the proof of the Frobenius descent [LS-Q2, 5.5] fails because it uses a proposition [LS-Q2, 5.2] depending on the local freeness of each term of the jet complex. In Subsection 4.2, we correct the proof of the Frobenius descent by using our higher de Rham complex.

\subsection{Conventions.}

Throughout this article, we fix a prime number $p$ and a natural number $m$ (natural number means, in this article, non-negative integer).

We assume that $p$ is nilpotent on all schemes appearing in this article.

If $k, k^{\prime}$ and $k^{\prime \prime}$ denote natural numbers such that $k=k^{\prime}+k^{\prime \prime}$, we often use the notation

$$
\left(\begin{array}{c}
k \\
k^{\prime}
\end{array}\right):=\frac{k !}{k^{\prime} ! k^{\prime \prime} !}, \quad\left\{\begin{array}{c}
k \\
k^{\prime}
\end{array}\right\}:=\frac{q !}{q^{\prime} ! q^{\prime \prime} !} \quad \text { and } \quad\left\langle\begin{array}{c}
k \\
k^{\prime}
\end{array}\right):=\left(\begin{array}{c}
k \\
k^{\prime}
\end{array}\right)\left\{\begin{array}{c}
k \\
k^{\prime}
\end{array}\right\}^{-1},
$$

where $q$ (resp. $q^{\prime}, q^{\prime \prime}$ ) denotes the integer part of $k / p^{m}$ (resp. $\left.k^{\prime} / p^{m}, k^{\prime \prime} / p^{m}\right)$. We also use the usual conventions on multi-indices; if $I=\left(i_{1}, \ldots, i_{n}\right)$ and $J=\left(j_{1}, \ldots, j_{n}\right)$ satisfies $J \leq I$, that is, if $j_{k} \leq i_{k}$ for all $k=1, \ldots, n$, then we define

$$
\left(\begin{array}{l}
I \\
J
\end{array}\right):=\prod_{k=1}^{n}\left(\begin{array}{l}
i_{k} \\
j_{k}
\end{array}\right), \quad\left\{\begin{array}{l}
I \\
J
\end{array}\right\}:=\prod_{k=1}^{n}\left\{\begin{array}{l}
i_{k} \\
j_{k}
\end{array}\right\} \quad \text { and }\left\langle\begin{array}{l}
I \\
J
\end{array}\right\rangle:=\prod_{k=1}^{n}\left\langle\begin{array}{l}
i_{k} \\
j_{k}
\end{array}\right\rangle .
$$

The element $(0, \ldots, 0,1,0, \ldots, 0)$ in $\mathbb{N}^{n}$, where 1 sits in the $i$-th entry, is denoted by $\mathbf{l}_{i}$.

\subsection{Acknowledgements.}

The author would like to express his greatest gratitude to Professor Atsushi Shiho for introducing me to the field of crystalline theory of higher level, reading thoroughly the draft of this paper, pointing out a lot of mistakes on it and giving him a lot of advice.

The author had a chance to talk with Professor Bernard Le Stum on this topic during his stay in Japan. The author is grateful to him for the meaningful argument. The author is also grateful to Professor Pierre Berthelot for his sincere and detailed answer to my questions on the crystalline site of higher level.

\section{Crystalline Site of Higher Level.}

\section{$1.1 m$-PD structures.}

First, let us recall some basic notions on $m$-PD structures. The fundamental reference on this subject is Berthelot's article [B3].

Definition 1.1.- Let $R$ be a $\mathbb{Z}_{(p)}$-algebra and $\mathfrak{a}$ an ideal of $R$. An $m$-PD structure on $\mathfrak{a}$ is a PD ideal $(\mathfrak{b}, \gamma)$ of $R$ that satisfies the following two conditions:

(a) $\mathfrak{a}^{\left(p^{m}\right)}+p \mathfrak{a} \subset \mathfrak{b} \subset \mathfrak{a}$;

(b) the PD structure $\gamma$ is compatible with the unique one on $p \mathbb{Z}_{(p)}$. 
Here, $\mathfrak{a}^{\left(p^{m}\right)}$ denotes the ideal of $R$ generated by $x^{p^{m}}$ for all elements $x$ of $\mathfrak{a}$. We call $(\mathfrak{a}, \mathfrak{b}, \gamma)$ an $m$-PD ideal of $R$, and $(R, \mathfrak{a}, \mathfrak{b}, \gamma)$ an $m$-PD ring.

An $m$-PD morphism $\left(R^{\prime}, \mathfrak{a}^{\prime}, \mathfrak{b}^{\prime}, \gamma^{\prime}\right) \rightarrow(R, \mathfrak{a}, \mathfrak{b}, \gamma)$ between two $m$-PD rings is a PD homomorphism $\left(R^{\prime}, \mathfrak{b}^{\prime}, \gamma^{\prime}\right) \rightarrow(R, \mathfrak{b}, \gamma)$ such that the image of $\mathfrak{a}^{\prime}$ lies in $\mathfrak{a}$.

Definition 1.2.- Let $(R, \mathfrak{a}, \mathfrak{b}, \gamma)$ be an $m$-PD ring and $A$ an $R$-algebra. We agree that $\widetilde{\mathfrak{b}}$ denotes the ideal $\mathfrak{b}+p R$, and that $\widetilde{\gamma}$ denotes the PD structure on $\widetilde{\mathfrak{b}}$ compatible with $\gamma$ and the unique one on $p \mathbb{Z}_{(p)}$.

(i) We say that the $m$-PD structure $(\mathfrak{b}, \gamma)$ extends to $A$ if the PD structure $\widetilde{\gamma}$ on $\widetilde{\mathfrak{b}}$ extends to $A$.

(ii) Let $(I, J, \delta)$ be an $m$-PD ideal of $A$. We say that the $m$-PD structure $(J, \delta)$ is compatible with $(\mathfrak{b}, \gamma)$ if the following two conditions hold:

(a) the two PD structures $\tilde{\gamma}$ and $\delta$ are compatible;

(b) $\widetilde{\mathfrak{b}} A \cap I$ is a PD sub-ideal of $\widetilde{\mathfrak{b}} A$ (equipped with the PD structure extending $\widetilde{\gamma}$ ).

(iii) Let $\left(\mathfrak{a}^{\prime}, \mathfrak{b}^{\prime}, \gamma^{\prime}\right)$ be another $m$-PD ideal of $R$. Then, this is called an $m-P D s u b$ ideal of $(\mathfrak{a}, \mathfrak{b}, \gamma)$ (or simply of $\mathfrak{a}$ ) if $\mathfrak{a}^{\prime}$ is a sub-ideal of $\mathfrak{a}$ and if $\left(\mathfrak{b}^{\prime}, \gamma^{\prime}\right)$ is a PD sub-ideal of $(\mathfrak{b}, \gamma)$.

DEFINITION 1.3.- Let $(R, \mathfrak{a}, \mathfrak{b}, \gamma)$ be an $m$-PD ring. For each natural number $k$ and element $x$ in $\mathfrak{a}$, we put

$$
x^{\{k\}}:=x^{r} \gamma_{q}\left(x^{p^{m}}\right),
$$

where $k=p^{m} q+r$ and $0 \leq r<p^{m}$.

As is easily seen, this function satisfies $q ! x^{\{k\}}=x^{k}$. Moreover, there are relations [B3, 1.3.6] similar to those of the classical divided power function; here, we recall here a relation [B3, 1.3.6 (iii)] that is used later.

Proposition 1.4.- Let $(R, \mathfrak{a}, \mathfrak{b}, \gamma)$ be an $m-P D$ ring. Then, for all $x, y \in \mathfrak{a}$ and $k \in \mathbb{N}$, we have

$$
(x+y)^{\{k\}}=\sum_{k^{\prime}=0}^{k}\left\langle\begin{array}{c}
k \\
k^{\prime}
\end{array}\right) x^{\left\{k^{\prime}\right\}} y^{\left\{k-k^{\prime}\right\}} .
$$

The following proposition, whose proof [B3, 1.4.1] we do not recall here, generalizes the notion of PD envelope.

Proposition-Definition 1.5.- Let $(R, \mathfrak{a}, \mathfrak{b}, \gamma)$ be an m-PD ring. Let $\mathscr{C}_{1}$ denote the category of the $m-P D$ rings over $R$ whose $m$-PD structure is compatible with $(\mathfrak{b}, \gamma)$, and $\mathscr{C}_{2}$ the category of the pairs $(A, I)$ consisting of an R-algebra A and an ideal I of $A$. Then, the forgetful functor $\mathscr{C}_{1} \rightarrow \mathscr{C}_{2}$ has a left adjoint functor. When $(A, I)$ is an object of $\mathscr{C}_{2}$, its image under this functor is denoted by $\left(D_{A, \gamma}^{(m)}(I), \bar{I}_{,}, \bar{I}_{0},{ }^{\left[{ }^{[}\right.}\right)$and is called the $m$-PD envelope of $(A, I)$ (compatible with $(\mathfrak{b}, \gamma)$ ).

\subsection{Crystalline site of level $m$.}

All the arguments in the previous subsection are obviously generalized to the schemetheoretical situation. 
Let $(S, \mathfrak{a}, \mathfrak{b}, \gamma)$ be an $m$-PD scheme, that is, a datum which consists of a scheme $S$, a quasi-coherent ideal $\mathfrak{a}$ of $\mathscr{O}_{S}$ and a quasi-coherent $m$-PD structure $(\mathfrak{b}, \gamma)$ on $\mathfrak{a}$. Given two $S$-schemes $X, Y$ to which the $m$-PD structure $(\mathfrak{b}, \gamma)$ extends, and given an immersion $X \hookrightarrow Y$ over $S$, we let $D_{X, \gamma}^{(m)}(Y)$ denote the $m$-PD envelope of the immersion.

Now, we fix throughout this subsection an $S$-scheme $X$, and assume that the $m$-PD structure $(\mathfrak{b}, \gamma)$ extends to $\mathscr{O}_{X}$. We recall the definition of $m$-crystalline site [LS-Q1, 4].

Definition 1.6.- (i) Let $U$ be an open subscheme of $X$. An $m$-PD thickening $(U, T, J, \delta)$ of $U$ over $(S, \mathfrak{a}, \mathfrak{b}, \gamma)$ is a datum which consists of an $S$-scheme $T$, a closed $S$-immersion $U \hookrightarrow T$ and an $m$-PD structure $(J, \delta)$ on the ideal of $U \hookrightarrow T$ compatible with $(\mathfrak{b}, \gamma)$.

(ii) The $m$-crystalline site $\operatorname{Cris}^{(m)}(X / S, \mathfrak{a}, \mathfrak{b}, \gamma)$, or $\operatorname{Cris}^{(m)}(X / S)$, is the category of the $m$-PD thickenings $(U, T, J, \delta)$ of an open subscheme $U$ of $X$ over $(S, \mathfrak{a}, \mathfrak{b}, \gamma)$, morphisms defined in an obvious way, the topology being defined so that a family $\left\{\left(U_{\lambda}, T_{\lambda}, J_{\lambda}, \delta_{\lambda}\right) \rightarrow(U, T, J, \delta)\right\}_{\lambda}$ of morphisms is a covering if and only if $\left\{T_{\lambda} \rightarrow\right.$ $T\}_{\lambda}$ is a covering with respect to the Zariski topology on $T$. Its associated topos $(X / S, \mathfrak{a}, \mathfrak{b}, \gamma)_{\text {cris }}^{(m)}$, or $(X / S)_{\text {cris }}^{(m)}$, is called the $m$-crystalline topos.

(iii) The sheaf of rings

$$
(U, T, J, \delta) \mapsto \Gamma\left(T, \mathscr{O}_{T}\right)
$$

in the topos $(X / S)_{\text {cris }}^{(m)}$ is called the structure sheaf of the site $\operatorname{Cris}^{(m)}(X / S)$, and denoted by $\mathscr{O}_{X /(S, \mathfrak{a}, \mathfrak{b}, \gamma)}^{(m)}$ or by $\mathscr{O}_{X / S}^{(m)}$.

For a sheaf $E \in(X / S)_{\text {cris }}^{(m)}$ and an object $(U, T, J, \delta)$ of $\operatorname{Cris}^{(m)}(X / S)$, we let $E_{(U, T, J, \delta)}$ denote the sheaf on $T$ induced by $E$. The most frequent situation is $U=T=X$; in this case, we simply write $E_{X}$ instead of $E_{(U, T, J, \delta)}$.

The $m$-crystalline topos has the functoriality which generalizes that of crystalline topos.

Proposition 1.7.- Let $u:\left(S^{\prime}, \mathfrak{a}^{\prime}, \mathfrak{b}^{\prime}, \gamma^{\prime}\right) \rightarrow(S, \mathfrak{a}, \mathfrak{b}, \gamma)$ be an $m$-PD morphism from another $m$-PD scheme, let $X^{\prime}$ be an $S^{\prime}$-scheme such that the m-PD structure $\left(\mathfrak{b}^{\prime}, \gamma^{\prime}\right)$ extends to $\mathscr{O}_{X^{\prime}}$, and let $g: X^{\prime} \rightarrow X$ be a morphism over $S$. The structure morphism of the $S$-scheme $X$ (resp. $S^{\prime}$-scheme $X^{\prime}$ ) is denoted by $f$ (resp. $f^{\prime}$ ).

Then, there exists a morphism of topoi

$$
g_{\text {cris }}^{(m)}:\left(X^{\prime} / S^{\prime}\right)_{\text {cris }}^{(m)} \rightarrow(X / S)_{\text {cris }}^{(m)} \text {. }
$$

We recall here the construction of this morphism of topoi. We firstly define a functor

$$
g^{*}: \operatorname{Cris}^{(m)}(X / S, \mathfrak{a}, \mathfrak{b}, \gamma) \rightarrow\left(X^{\prime} / S^{\prime}, \mathfrak{a}^{\prime}, \mathfrak{b}^{\prime}, \gamma^{\prime}\right)_{\text {cris }}^{(m)}
$$

as follows: for an object $(U, T, J, \delta)$ of $\operatorname{Cris}^{(m)}(X / S)$, the sheaf $g^{*}(U, T, J, \delta)$ sends each object $\left(U^{\prime}, T^{\prime}, J^{\prime}, \delta^{\prime}\right)$ of Cris ${ }^{(m)}\left(X^{\prime} / S^{\prime}\right)$ to the set of $m$-PD morphisms $T^{\prime} \rightarrow T$ over $g$; here, an " $m$-PD morphism over $g$ " is defined to be a morphism $h: T^{\prime} \rightarrow T$ that coincides with $g$ on $U^{\prime}$, that induces a morphism of closed immersions $\left(U^{\prime} \hookrightarrow T^{\prime}\right) \rightarrow$ 
$(U \hookrightarrow T)$ and that is an $m$-PD morphism with respect to the $m$-PD structures $\left(J^{\prime}, \delta^{\prime}\right)$ and $(J, \delta)$.

The functor $g^{*}$ is continuous, the topology of the target being the canonical topology; the classical proof [B1, III 2.2.2] works verbatim. This shows that $g^{*}$ extends to the functor

$$
g_{\text {cris }}^{(m)^{*}}:(X / S, \mathfrak{a}, \mathfrak{b}, \gamma)_{\text {cris }}^{(m)} \rightarrow\left(X^{\prime} / S^{\prime}, \mathfrak{a}^{\prime}, \mathfrak{b}^{\prime}, \gamma^{\prime}\right)_{\text {cris }}^{(m)}
$$

that admits a right adjoint $g_{\text {cris * }}^{(m)}$ [SGA4, III 1.2]. We may follow the classical argument [B1, III 2.2] to show that the functor $g_{\text {cris }}^{(m)}$ is left exact, which gives the morphism of topoi $g_{\text {cris }}^{(m)}=\left(g_{\text {cris }}^{(m)^{*}}, g_{\text {cris * }}^{(m)}\right)$.

Before closing this section, we recall another fundamental morphism

$$
u_{X / S}^{(m)}:(X / S)_{\text {cris }}^{(m)} \rightarrow X_{\text {Zar }}
$$

of topoi, which we call the projection of $m$-crystalline topos to the Zariski topos. For a sheaf $E$ on $X$, we define the sheaf $u_{X / S}^{(m)}{ }^{*}(E)$ on $\operatorname{Cris}^{(m)}(X / S)$ to be the sheaf that sends $(U, T, J, \delta)$ to $E(U)$; we get a functor $u_{X / S}^{(m)}{ }^{*}: X_{\mathrm{Zar}} \rightarrow(X / S)_{\text {cris }}^{(m)}$. This functor is exact and has a right adjoint $u_{X / S *}^{(m)}:(X / S)_{\text {cris }}^{(m)} \rightarrow X_{\mathrm{Zar}}$, described as in the classical case [B1, III 3.2]. Now, we get a morphism $u_{X / S}=\left(u_{X / S}^{(m)}{ }^{*}, u_{X / S *}^{(m)}\right)$ of topoi.

At last, letting $f: X \rightarrow S$ denote the structure morphism, we define the morphism

$$
f_{X / S}^{(m)}:(X / S)_{\text {cris }}^{(m)} \rightarrow S_{\text {Zar }}
$$

to be the composite of $u_{X / S}^{(m)}$ and the morphism $f_{\mathrm{Zar}}: X_{\mathrm{Zar}} \rightarrow S_{\mathrm{Zar}}$.

\subsection{Crystals, differential operators and stratifications.}

In this subsection, we discuss the notion of $m$-crystal, hyper $m$-PD differential operator and hyper $m$-PD stratification. Let $(S, \mathfrak{a}, \mathfrak{b}, \gamma)$ be an $m$-PD scheme, and $X$ an $S$-scheme such that the $m$-PD structure $(\mathfrak{b}, \gamma)$ extends to $\mathscr{O}_{X}$. Recall that $p$ is nilpotent on $S$ as we have assumed in Subsection 0.3.

Definition 1.8.- Let $E$ be an $\mathscr{O}_{X / S}^{(m)}$-module in $(X / S)_{\text {cris }}^{(m)}$. Then, $E$ is called an $m$ crystal in $\mathscr{O}_{X / S}^{(m)}$-modules if for all morphism $f:(U, T, J, \delta) \rightarrow\left(U^{\prime}, T^{\prime}, J^{\prime}, \delta^{\prime}\right)$ of Cris ${ }^{(m)}(X / S)$, the canonical morphism

$$
f^{*}\left(E_{\left(U^{\prime}, T^{\prime}, J^{\prime}, \delta^{\prime}\right)}\right) \rightarrow E_{(U, T, J, \delta)}
$$

is an isomorphism.

In this article, $P_{X / S}^{(m)}$ denotes the $m$-PD envelope of the diagonal immersion $X \hookrightarrow$ $X \times_{S} X$, and $\mathscr{P}_{X / S}^{(m)}$ denotes the structure sheaf of $P_{X / S}^{(m)}$. We always regard $X \times_{S} X$ and $P_{X / S}^{(m)}$ as schemes over $X$ by the first projection; the sheaf $\mathscr{O}_{X}$, therefore, acts on $\mathscr{O}_{X \times{ }_{S} X}=\mathscr{O}_{X} \otimes_{\mathscr{O}_{S}} \mathscr{O}_{X}$ by multiplication to the first factor, and this also makes $\mathscr{P}_{X / S}^{(m)}$ an $\mathscr{O}_{X}$-algebra.

Definition 1.9.- Let $M$ and $N$ be two $\mathscr{O}_{X}$-modules. Then, a hyper $m$-PD differ- 
ential operator from $M$ to $N$ is an $\mathscr{O}_{X}$-linear morphism

$$
\mathscr{P}_{X / S}^{(m)} \otimes \mathscr{O}_{X} M \rightarrow N
$$

DEFINITION 1.10.- Let $M$ be an $\mathscr{O}_{X}$-module. Then, a hyper $m$-PD stratification on $M$ is a $\mathscr{P}_{X / S}^{(m)}$-linear isomorphism

$$
\mathscr{P}_{X / S}^{(m)} \otimes_{\mathscr{O}_{X}} M \rightarrow M \otimes_{\mathscr{O}_{X}} \mathscr{P}_{X / S}^{(m)}
$$

that induces the identity map on $M$ after passing the quotient $\mathscr{P}_{X / S}^{(m)} \rightarrow \mathscr{O}_{X}$, and that satisfies the usual cocycle condition.

Now, let $\left(\mathfrak{a}_{0}, \mathfrak{b}_{0}, \gamma_{0}\right)$ be a quasi-coherent $m$-PD sub-ideal of $\mathfrak{a}$, let $S_{0} \hookrightarrow S$ denote the closed immersion defined by $\mathfrak{a}_{0}$, and let $i: X_{0} \hookrightarrow X$ denote its base change by $X \rightarrow S$. We assume that $X$ is smooth over $S$.

The first important proposition in this situation is the following one.

PROPOSITION 1.11.- In the situation above, the functor

$$
i_{\text {cris } *}^{(m)}:\left(X_{0} / S\right)_{\text {cris }}^{(m)} \rightarrow(X / S)_{\text {cris }}^{(m)}
$$

is exact, where $i_{\text {cris } *}^{(m)}$ is the direct image functor of the morphism $i_{\text {cris }}^{(m)}$ of topoi induced from $i$ (Proposition 1.7). Moreover, the image of the structure sheaf $\mathscr{O}_{X_{0} / S}^{(m)}$ under this functor is isomorphic to $\mathscr{O}_{X / S}^{(m)}$, and the image of an $m$-crystal in $\mathscr{O}_{X_{0} / S}^{(m)}$-modules is an m-crystal in $\mathscr{O}_{X / S}^{(m)}$-modules.

Proof. If $(U, T, J, \delta)$ is an $m$-PD thickening in $\operatorname{Cris}^{(m)}(X / S)$, and if $U_{0}$ denotes the fiber product $U \times_{X} X_{0}$, then the closed immersion $U_{0} \hookrightarrow T$ defines an $m$-PD thickening, which is an object of $\operatorname{Cris}^{(m)}\left(X_{0} / S\right)$. Let $\left(U_{0}, T\right)$ denote this thickening. Then, $\left(U_{0}, T\right)$ represents $i^{*}\left(U_{0}, T\right)$. Because of the construction and of Yoneda's lemma, if $E$ is an $\mathscr{O}_{X_{0} / S}^{(m)}$-module, $i_{\text {cris,* }}(E)(U, T)$ is canonically isomorphic to $E\left(U_{0}, T\right)$, which gives a canonical isomorphism

$$
i_{\text {cris } *}^{(m)}(E)_{(U, T, J, \delta)} \stackrel{\sim}{\longrightarrow} E_{\left(U_{0}, T\right)} .
$$

All assertions in the proposition follow from this isomorphism.

REMARK 1.12.- (This remark is due to P. Berthelot [B5].)

In case $m=0$, Proposition 1.11 holds for an arbitrary closed immersion $i: X_{0} \rightarrow$ $Y$ over $S$ to a smooth $S$-scheme $Y$. If $m>0$, however, the direct image of an $m$ crystal along such a closed immersion is not necessarily an $m$-crystal. This defect can be avoided if we work in the higher-level analogue of the restricted crystalline site [B1, IV.2].

In this article, we do not introduce the "restricted $m$-crystalline site" because we do not treat arbitrary closed immersions.

The following proposition and the corollary is proved as in the classical case [B-O, 6.6,6.7] with the aid of Proposition 1.11. 
Proposition 1.13.- In the situation above, the following categories are equivalent:

(i) the category of the $m$-crystals in $\mathscr{O}_{X_{0} / S}^{(m)}$-modules;

(ii) the category of the $\mathscr{O}_{X}$-modules equipped with a hyper $m$-PD stratification.

Proof. For the sake of completeness of the article, we include here a construction of the equivalence of these categories following the classical one $[\mathrm{B}-\mathrm{O}, 6.6]$ applied in our closed immersion.

First, let $E$ be an $m$-crystal. Then, the two projection $p_{1}, p_{2}: P_{X / S}^{(m)} \rightarrow X$ give isomorphisms $p_{i}^{*} E_{X} \rightarrow E_{P_{X / S}^{(m)}}(i=1,2)$. Then we get an isomorphism $\mathscr{P}_{X / S}^{(m)} \otimes_{\mathscr{O}_{X}} E_{X} \rightarrow$ $E_{X} \otimes_{\mathscr{O}_{X}} \mathscr{P}_{X / S}^{(m)}$, which gives a hyper $m$-PD stratification on the $\mathscr{O}_{X}$-module $E_{X}$.

Now, let $F$ be an $\mathscr{O}_{X}$-module equipped with a hyper $m$-PD stratification. We construct an $m$-crystal $E$ in $\mathscr{O}_{X_{0} / S}^{(m)}$-modules. For each object $(U, T, J, \delta)$ of $\operatorname{Cris}^{(m)}(X / S)$ with a retraction $h: T \rightarrow X$ extending $U \rightarrow X$, the sheaf $E_{(U, T, J, \delta)}$ is defined to be $h^{*}\left(\mathscr{O}_{F}\right)$; this condition defines a unique $m$-crystal $E$.

COROLlary 1.14.- The functor $i_{\text {cris* }}^{(m)}$ in Proposition 1.11 induces an equivalence of categories from the category of the $m$-crystals in $\mathscr{O}_{X_{0} / S}^{(m)}$-modules to that of the $m$ crystals in $\mathscr{O}_{X / S}^{(m)}$-modules. A quasi-inverse of this functor is the inverse image functor $i_{\text {cris }}^{(m)^{*}}$ of the morphism $i_{\text {cris }}^{(m)}$ of topoi induced from $i$.

\subsection{Linearization.}

Here, we discuss the linearization. Let $(S, \mathfrak{a}, \mathfrak{b}, \gamma)$ be an $m$-PD scheme, and $X$ an $S$ scheme such that the $m$-PD structure $(\mathfrak{b}, \gamma)$ extends to $\mathscr{O}_{X}$.

First, $j_{X}$ is the localization morphism

$$
j_{X}:\left.(X / S)_{\text {cris }}^{(m)}\right|_{X} \rightarrow(X / S)_{\text {cris }}^{(m)},
$$

where the source denotes the localized category of $(X / S)_{\text {cris }}^{(m)}$ over the $m$-PD thickening $(X, X, 0)$ with the trivial PD structure on 0 . Then, composing with $u_{X / S}^{(m)}$, we get the morphism of topoi

$$
\left.u_{X / S}^{(m)}\right|_{X}:\left.(X / S)_{\text {cris }}^{(m)}\right|_{X} \rightarrow X_{\text {Zar }} .
$$

Now, we define the linearization functor as

$$
L^{(m)}=\left.j_{X *} \circ u_{X / S}^{(m)}\right|_{X} ^{*}: X_{\mathrm{Zar}} \rightarrow(X / S)_{\text {cris }}^{(m)} .
$$

The $\mathscr{O}_{X}$-module $L^{(m)}(\mathscr{F})_{X}$ is also denoted by $L_{X}^{(m)}(\mathscr{F})$.

Proposition 1.15.- Assume that $X$ is smooth over $S$, and let $\mathscr{F}$ be an $\mathscr{O}_{X}$-module.

(i) $L^{(m)}(\mathscr{F})$ is an $m$-crystal, and $L_{X}^{(m)}(\mathscr{F})=\mathscr{P}_{X / S}^{(m)} \otimes_{\mathscr{O}_{X}} \mathscr{F}$.

(ii) We have $R u_{X / S *}^{(m)} L^{(m)}(\mathscr{F})=\mathscr{F}$.

(iii) If $E$ is an $m$-crystal, there exists a canonical isomorphism

$$
E \otimes_{\mathscr{O}_{X / S}^{(m)}} L^{(m)}(\mathscr{F}) \rightarrow L^{(m)}\left(E_{X} \otimes_{\mathscr{O}_{X}} \mathscr{F}\right) .
$$


Proof. By the construction of $L^{(m)}$, for each $(U, T, J, \delta) \in \operatorname{Cris}^{(m)}(X / S)$ we have

$$
L^{(m)}(\mathscr{F})_{(U, T, J, \delta)}=p_{T_{*}} p_{X}{ }^{*}(\mathscr{F}),
$$

where $p_{T}$ (resp. $p_{X}$ ) denotes the projection from $D_{U, \gamma}^{(m)}\left(T \times_{S} X\right)$ to $T$ (resp. to $X$ ). This, in particular, shows the latter half of (i).

As for the former half, by following the classical argument [B1, IV 3.1.6], it is sufficient to show that the natural morphism

$$
D_{U, \gamma}^{(m)}\left(T \times_{S} X\right) \rightarrow T \times{ }_{X} P_{X / S}^{(m)}
$$

is an isomorphism for all $m$-PD thickenings $(U, T, J, \delta)$ such that a retraction $T \rightarrow X$ exists. Now, because $\mathscr{P}_{X / S}^{(m)}$ is locally isomorphic to an $m$-PD polynomial algebra over $\mathscr{O}_{X}$, the $\mathscr{O}_{T}$-algebra $\mathscr{O}_{T} \otimes_{\mathscr{O}_{X}} \mathscr{P}_{X / S}^{(m)}$ is an $m$-PD polynomial algebra (over $\mathscr{O}_{T}$ ). Therefore, the ideal of the closed immersion $U \hookrightarrow T \times{ }_{X} P_{X / S}^{(m)}$ has an $m$-PD structure compatible with $\gamma$. Hence a morphism $T \times{ }_{X} P_{X / S}^{(m)} \rightarrow D_{U, \gamma}^{(m)}\left(T \times{ }_{S} X\right)$ is obtained, and the universality shows that this is an inverse morphism of (1.4.1).

The proof of (ii) and (iii) can be found in [LS-Q2, 4].

Now, we set some notations. For each natural number $r$, let $X_{/ S}^{(r)}$ denote the fiber product over $S$ of $r$ copies of $X$. The $m$-PD envelope of the diagonal immersion $X \hookrightarrow X_{/ S}^{(r+1)}$ is denoted by $P_{X / S}^{(m)}(r)$, and its structure sheaf is denoted by $\mathscr{P}_{X / S}^{(m)}(r)$. We have therefore $P_{X / S}^{(m)}=P_{X / S}^{(m)}(1)$ and $\mathscr{P}_{X / S}^{(m)}=\mathscr{P}_{X / S}^{(m)}(1)$. Again, we regard $X_{/ S}^{(r+1)}$ and $P_{X / S}^{(m)}(r)$ as schemes over $X$ by the first projection, which lets $\mathscr{O}_{X}$ act on $\mathscr{O}_{X_{I S}^{(r+1)}}=$ $\mathscr{O}_{X} \otimes_{\mathscr{O}_{S}} \cdots \otimes_{\mathscr{O}_{S}} \mathscr{O}_{X}$ by multiplication to the first factor and induce on $\mathscr{P}_{X / S}^{(m)}(r)$ an $\mathscr{O}_{X}$-algebra structure.

For each natural number $r$ and $i \in\{0, \ldots, r\}$, let $j_{r}^{i}: X_{I S}^{(r+1)} \rightarrow X$ denote the $(i+1)$ st projection. Let $d_{r}^{i}: P_{X / S}^{(m)}(r+1) \rightarrow P_{X / S}^{(m)}(r)(0 \leq i \leq r+1)$ denote the morphism corresponding to

$$
\left(j_{r+1}^{0}, \ldots, j_{r+1}^{i-1}, j_{r+1}^{i+1}, \ldots, j_{r+1}^{r+1}\right)_{S}: X_{/ S}^{(r+2)} \rightarrow X_{/ S}^{(r+1)},
$$

and $s_{r}^{i}: P_{X / S}^{(m)}(r-1) \rightarrow P_{X / S}^{(m)}(r)(0 \leq i \leq r)$ the one corresponding to

$$
\left(j_{r-1}^{0}, \ldots, j_{r-1}^{i}, j_{r-1}^{i}, \ldots, j_{r-1}^{r}\right)_{S}: X_{/ S}^{(r)} \rightarrow X_{/ S}^{(r+1)} .
$$

Then, these data make $P_{X / S}^{(m)}(\bullet)$ a simplicial scheme over $S$, and consequently $\mathscr{P}_{X / S}^{(m)}(\bullet)$ is a DGA (differential graded algebra) over $f^{-1}\left(\mathscr{O}_{S}\right)$; the differential morphism $d^{r}: \mathscr{P}_{X / S}^{(m)}(r) \rightarrow \mathscr{P}_{X / S}^{(m)}(r+1)$ is by definition

$$
d^{r}=\sum_{i=0}^{r+1}(-1)^{i} d_{r}^{i^{*}} .
$$

If $X$ is smooth over $S$, then $\mathscr{P}_{X / S}^{(m)}$ is isomorphic to the $m$-PD polynomial algebra $\mathscr{O}_{X}\left\{\tau_{1}, \ldots, \tau_{n}\right\}_{(m)}$. Moreover, the graded $\mathscr{O}_{X}$-algebra $\mathscr{P}_{X / S}^{(m)}(\bullet)$ can be identified with the tensor algebra of $\mathscr{P}_{X / S}^{(m)}$ over $\mathscr{O}_{X}$ [B3, Remarque after 2.1.3]; in particular, $\mathscr{P}_{X / S}^{(m)}(2)$ is isomorphic to $\mathscr{P}_{X / S}^{(m)} \otimes_{\mathscr{O}_{X}} \mathscr{P}_{X / S}^{(m)}$. 
Now, we proceed to a calculation of the hyper $m$-PD stratification on $\mathscr{P}_{X / S}^{(m)} \otimes_{\mathscr{O}_{X}}$ $\mathscr{F}=L_{X}^{(m)}(\mathscr{F})$, which exists because of Proposition 1.15 (i) and Proposition 1.13.

LEMMA 1.16.- Assume that $X$ is smooth over $S$. Then, the $m-P D$ stratification on $L_{X}^{(m)}(\mathscr{F})=\mathscr{P}_{X / S}^{(m)} \otimes_{\mathscr{O}_{X}} \mathscr{F}$ is induced by

$$
\begin{aligned}
& \mathscr{O}_{X_{/ S}^{(2)}} \otimes\left(\mathscr{O}_{X_{I S}^{(2)}} \otimes \mathscr{F}\right) \rightarrow\left(\mathscr{O}_{X_{I S}^{(2)}} \otimes \mathscr{F}\right) \otimes \mathscr{O}_{X_{/ S}^{(2)}} ; \\
& (1 \otimes 1) \otimes(f \otimes g) \otimes x \mapsto(1 \otimes g) \otimes x \otimes(1 \otimes f),
\end{aligned}
$$

where the tensor products are taken over $\mathscr{O}_{X}$.

Proof. By the proof of Proposition 1.13 and by Proposition 1.15 (i), the $m$-PD stratification on $L_{X}^{(m)}(\mathscr{F})$ is the composite of the isomorphism

$$
\mathscr{P}_{X / S}^{(m)} \otimes\left(\mathscr{P}_{X / S}^{(m)} \otimes \mathscr{F}\right) \rightarrow \mathscr{P}_{X / S}^{(m)}(2) \otimes \mathscr{F} ; \quad(1 \otimes 1) \otimes(f \otimes g) \otimes x \mapsto 1 \otimes f \otimes g \otimes x
$$

and the inverse of the isomorphism

$$
\left(\mathscr{P}_{X / S}^{(m)} \otimes \mathscr{F}\right) \otimes \mathscr{P}_{X / S}^{(m)} \rightarrow \mathscr{P}_{X / S}^{(m)}(2) \otimes \mathscr{F} ; \quad(1 \otimes 1) \otimes x \otimes(f \otimes g) \mapsto f \otimes g \otimes 1 \otimes x .
$$

Now, an easy observation shows the assertion.

We omit the proof of the following proposition since it is just a generalization of the classical argument.

Proposition 1.17.- Let $M$ and $N$ be two $\mathscr{O}_{X}$-modules, and $u$ a hyper $m$-PD differential operator from $M$ to $N$. Then, the morphism

$$
\mathscr{P}_{X / S}^{(m)} \otimes_{\mathscr{O}_{X}} M \stackrel{d_{1}^{1^{*} \otimes \mathrm{id}}}{\longrightarrow} \mathscr{P}_{X / S}^{(m)} \otimes_{\mathscr{O}_{X}} \mathscr{P}_{X / S}^{(m)} \otimes_{\mathscr{O}_{X}} M \stackrel{\mathrm{id} \otimes u}{\longrightarrow} \mathscr{P}_{X / S}^{(m)} \otimes_{\mathscr{O}_{X}} N
$$

is compatible with the hyper $m-P D$ stratifications on both sides viewed as $\mathscr{P}_{X / S}^{(m)} \otimes M=$ $L_{X}^{(m)}(M)$ and as $\mathscr{P}_{X / S}^{(m)} \otimes N=L_{X}^{(m)}(N)$.

\subsection{Differential calculus.}

We start with a general setting. Let $(S, \mathfrak{a}, \mathfrak{b}, \gamma)$ be an $m$-PD scheme and $f: X \rightarrow S$ a morphism such that the $m$-PD structure $(\mathfrak{b}, \gamma)$ extends to $\mathscr{O}_{X}$.

We define the sub-DGA $N \mathscr{P}_{X / S}^{(m)} \bullet$ by

$$
N \mathscr{P}_{X / S}^{(m), r}=\bigcap_{i=0}^{r+1} \operatorname{Ker}\left(s_{r}^{i^{*}}\right) .
$$

Now, we assume that the morphism $f: X \rightarrow S$ is smooth, and that $X$ has global coordinates $t=\left(t_{1}, \ldots, t_{n}\right)$ over $S$; we set $\tau_{i}:=d^{0}\left(t_{i}\right) \in N \mathscr{P}_{X / S}^{(m), 1}$, which is the pullback of $t_{i} \otimes 1-1 \otimes t_{i}$ by the natural morphism $P_{X / S}^{(m)} \rightarrow X \times_{S} X$; for $I=\left(i_{1}, \ldots, i_{n}\right) \in \mathbb{N}^{n}$, we set

$$
\tau^{\{I\}}:=\prod_{j=1}^{n} \tau_{j}^{\left\{i_{j}\right\}} .
$$


Under this notation, $N \mathscr{P}_{X / S}^{(m), 1}$ is freely generated by $\left\{\tau^{\{I\}}\right\}_{I \in \mathbb{N}^{n} \backslash\{0\}}$, and the DGA $N \mathscr{P}_{X / S}^{(m), \bullet}$ is isomorphic to the tensor algebra of $N \mathscr{P}_{X / S}^{(m), 1}$ over $\mathscr{O}_{X}$.

We keep these assumptions and notations in the remainder of this subsection. fies

Proposition 1.18.- For all $I \in \mathbb{N}^{n}$, the morphism $d^{1}: \mathscr{P}_{X / S}^{(m)}(1) \rightarrow \mathscr{P}_{X / S}^{(m)}(2)$ satis-

$$
d^{1}\left(\tau^{\{I\}}\right)=-\sum_{0<V<I}\left\langle\begin{array}{l}
I \\
V
\end{array}\right\rangle \tau^{\{V\}} \otimes \tau^{\{I-V\}}
$$

Proof. Let us recall that $d^{1}$ is by definition equal to $d_{1}^{0 *}-d_{1}^{1 *}+d_{1}^{2 *}$. We have

$$
d_{1}^{0 *}\left(\tau^{\{l\}}\right)=\tau^{\{l\}} \otimes 1 \text { and } d_{1}^{2^{*}}\left(\tau^{\{l\}}\right)=1 \otimes \tau^{\{l\}} .
$$

By using the equation $d_{1}^{1^{*}}\left(\tau_{i}\right)=\tau_{i} \otimes 1+1 \otimes \tau_{i}$ and Proposition 1.4, we calculate

$$
\begin{aligned}
d_{1}^{1^{*}}\left(\tau^{\{I\}}\right)=(\tau \otimes 1+1 \otimes \tau)^{\{I\}} & =\sum_{0 \leq V \leq I}\left\langle\begin{array}{c}
I \\
V
\end{array}\right\rangle(\tau \otimes 1)^{\{V\}}(1 \otimes \tau)^{\{I-V\}} \\
& =\sum_{0 \leq V \leq I}\left\langle\begin{array}{c}
I \\
V
\end{array}\right\rangle \tau^{\{V\}} \otimes \tau^{\{I-V\}} .
\end{aligned}
$$

This completes the proof.

The hyper $m$-PD stratification on $\mathscr{P}_{X / S}^{(m)}=L_{X}^{(m)}\left(\mathscr{O}_{X}\right)$ is described in the following proposition.

Proposition 1.19.- The hyper m-PD stratification

$$
\mathscr{P}_{X / S}^{(m)} \otimes_{\mathscr{O}_{X}} \mathscr{P}_{X / S}^{(m)} \rightarrow \mathscr{P}_{X / S}^{(m)} \otimes_{\mathscr{O}_{X}} \mathscr{P}_{X / S}^{(m)}
$$

of $\mathscr{P}_{X / S}^{(m)}$ maps $1 \otimes \tau^{\{I\}}$ to

$$
\sum_{0 \leq V \leq I}\left\langle\begin{array}{c}
I \\
V
\end{array}\right\rangle \tau^{\{V\}} \otimes(-\tau)^{\{I-V\}} .
$$

Proof. By Lemma 1.16, the hyper $m$-PD stratification is induced by

$$
\mathscr{O}_{X_{/ S}^{(2)}} \otimes_{\mathscr{O}_{X}} \mathscr{O}_{X_{/ S}^{(2)}} \rightarrow \mathscr{O}_{X_{/ S}^{(2)}} \otimes_{\mathscr{O}_{X}} \mathscr{O}_{X_{I S}^{(2)}} ; \quad(1 \otimes 1) \otimes(f \otimes g) \mapsto(1 \otimes g) \otimes(1 \otimes f) .
$$

Noting that $\left(t_{i} \otimes 1\right) \otimes(1 \otimes 1)=(1 \otimes 1) \otimes\left(t_{i} \otimes 1\right)$, we see that this morphism sends $(1 \otimes$ $1) \otimes\left(t_{i} \otimes 1-1 \otimes t_{i}\right)$ to

$$
\left(t_{i} \otimes 1-1 \otimes t_{i}\right) \otimes(1 \otimes 1)-(1 \otimes 1) \otimes\left(t_{i} \otimes 1-1 \otimes t_{i}\right),
$$

therefore the hyper $m$-PD stratification sends $1 \otimes \tau^{\{l\}}$ to

$$
(\tau \otimes 1-1 \otimes \tau)^{\{l\}} \text {. }
$$

Now, we may calculate as in the proof of Proposition 1.18 to complete the proof.

Similarly, starting from the simplicial scheme $P_{X / S}^{(m)}(\bullet+1)$ over $X$, we construct a 
DGA $L \mathscr{P}_{X / S}^{(m)}(\bullet):=P_{X / S}^{(m)}(\bullet+1)$ over $\mathscr{O}_{X}$ and its sub-DGA

$$
L N \mathscr{P}_{X / S}^{(m), \bullet}:=\bigcap_{i=1}^{r+2} \operatorname{Ker}\left(s_{r+1}^{i}{ }^{*}\right)
$$

Note that the differential morphism $d^{r}: L_{X / S}^{(m)}(r) \rightarrow L \mathscr{P}_{X / S}^{(m)}(r+1)$ is by definition

$$
d^{r}=\sum_{i=1}^{r+2}(-1)^{i+1} d_{r+1}^{i}{ }^{*}
$$

By Proposition 1.15 , each $L \mathscr{P}_{X / S}^{(m)}(r)=L_{X}^{(m)}\left(\mathscr{P}_{X / S}^{(m)}(r)\right)$ has a hyper $m$-PD stratification.

LEMMA 1.20.- For each natural number $r$, the differential morphism $d^{r}: \mathscr{P}_{X / S}^{(m)}(r) \rightarrow$ $L \mathscr{P}_{X / S}^{(m)}(r+1)$ is compatible with the hyper $m-P D$ stratification on both sides.

Proof. By Lemma 1.16, the hyper $m$-PD stratification on $L \mathscr{P}_{X / S}^{(m)}(r)$ is induced by

$$
\begin{gathered}
\mathscr{O}_{X_{I S}^{(2)}} \otimes_{\mathscr{O}_{X}} \mathscr{O}_{X_{I S}^{(r+2)}} \longrightarrow \mathscr{O}_{X_{I S}^{(r+2)}} \otimes_{\mathscr{O}_{X}} \mathscr{O}_{X_{I S}^{(2)}} \\
(1 \otimes 1) \otimes\left(f \otimes g \otimes h_{1} \otimes \cdots \otimes h_{r}\right) \longmapsto\left(1 \otimes g \otimes h_{1} \otimes \cdots \otimes h_{r}\right) \otimes(1 \otimes f) .
\end{gathered}
$$

The morphism $d_{r+1}^{i}{ }^{*}$ for $i=1, \ldots, r+1$ is therefore compatible with this morphism, and so is $d^{r}$ because of its definition (1.5.3).

\section{Higher Poincaré Lemma - Local Results.}

\subsection{Higher de Rham complex.}

Throughout Section 2, we fix an $m$-PD scheme $(S, \mathfrak{a}, \mathfrak{b}, \gamma)$ and a smooth $S$-scheme $X$ that has global coordinates $t=\left(t_{1}, \ldots, t_{n}\right)$; precisely, we assume that there exists an étale morphism $g: X \rightarrow \mathbb{A}_{S}^{n}=\operatorname{Spec} \mathscr{O}_{S}\left[t_{1}^{\prime}, \ldots, t_{n}^{\prime}\right]$ over $S$ and we write the $t_{i}=g^{*}\left(t_{i}^{\prime}\right)$ for $i=1, \ldots, n$.

First, in this subsection, we introduce the "higher de Rham complex" in this situation. Recall from (1.5.1) and (1.5.2) the definition of $N \mathscr{P}_{X / S}^{(m), \bullet}$ and $L N \mathscr{P}_{X / S}^{(m), \bullet}$

Let $\mathscr{K}_{X / S}^{(m), \bullet}$ be the DG-ideal of $N \mathscr{P}_{X / S}^{(m), \bullet}$ generated by $\tau^{\{I\}} \in N \mathscr{P}_{X / S}^{(m), 1}$, s, where $I$ runs through all multi-indices in $\mathbb{N}^{n} \backslash\left\{0, p^{m} \mathbf{1}_{1}, \ldots, p^{m} \mathbf{1}_{n}\right\}$. It is easy to show that the ideal $\mathscr{P}_{X / S}^{(m)} \otimes \mathscr{K}_{X / S}^{(m), \bullet}$ of $L N \mathscr{P}_{X / S}^{(m), \bullet}$ is a DG-ideal.

Definition 2.1.- The higher de Rham complex $\breve{\Omega}_{X / S}^{(m), \bullet}$ is by definition the quotient of $N \mathscr{P}_{X / S}^{(m), \bullet}$ by the DG-ideal $\mathscr{K}_{X / S}^{(m), \bullet}$ above.

The linearized higher de Rham complex $L \breve{\Omega}_{X / S}^{(m), \bullet}$ is by definition the quotient of $L N \mathscr{P}_{X / S}^{(m)}$ by $\mathscr{P}_{X / S}^{(m)} \otimes \mathscr{K}_{X / S}^{(m), \bullet}$.

It should be remarked that these complexes essentially depend on the choice of the system of global coordinates on $X$. This construction therefore can not be generalized to the global situation. 
For $i=1, \ldots, n$, the image of $\tau_{i}^{p^{m}}$ under the natural surjection $N \mathscr{P}_{X / S}^{(m), 1} \rightarrow \breve{\Omega}_{X / S}^{(m), 1}$ is denoted by $\bar{\tau}_{i}^{p^{m}}$.

Proposition 2.2.- (i) $\breve{\Omega}_{X / S}^{(m), 1}$ is a free $\mathscr{O}_{X}$-module of rank $n$ with basis $\left\{\bar{\tau}_{j}^{p^{m}}\right\}_{j=1, \ldots, n}$.

(ii) $\breve{\Omega}_{X / S}^{(m) \cdot \bullet}$ is isomorphic to the exterior algebra of $\breve{\Omega}_{X / S}^{(m), 1}$ as a graded $\mathscr{O}_{X}$-module.

(iii) The differential map $d^{r}$ sends the section $\tau^{\{I\}} \otimes \bar{\tau}_{j_{1}}^{p^{m}} \wedge \cdots \wedge \bar{\tau}_{j_{r}}^{p^{m}}$ of $L \breve{\Omega}_{X / S}^{(m), r}$, where $I=\left(i_{1}, \ldots, i_{n}\right) \in \mathbb{N}^{n}$, to the section

$$
\sum_{\substack{j=1, \ldots, n \\
i_{j} \geq p^{m}}}\left\langle\begin{array}{c}
i_{j} \\
p^{m}
\end{array}\right\rangle \tau^{\left\{I-p^{m} \mathbf{1}_{j}\right\}} \otimes \bar{\tau}_{j}^{p^{m}} \wedge \bar{\tau}_{j_{1}}^{p^{m}} \wedge \cdots \wedge \bar{\tau}_{j_{r}}^{p^{m}} .
$$

Proof. The $\mathscr{O}_{X}$-module $\breve{\Omega}_{X / S}^{(m), r}$ is generated by the sections

$$
\bar{\tau}_{j_{1}}^{p^{m}} \otimes \bar{\tau}_{j_{2}}^{p^{m}} \otimes \cdots \otimes \bar{\tau}_{j_{r}}^{p^{m}},
$$

and their relations are generated by

$$
\sum_{\substack{0<V<I \\
V=p^{m} \mathbf{1}_{i}, I-V=p^{m} \mathbf{1}_{j}}}\left\langle\begin{array}{c}
I \\
V
\end{array}\right\rangle \bar{\tau}_{i}^{p^{m}} \otimes \bar{\tau}_{j}^{p^{m}}=0 .
$$

For $I=2 p^{m} \mathbf{1}_{i}$, this gives

$$
\bar{\tau}_{i}^{p^{m}} \otimes \bar{\tau}_{i}^{p^{m}}=0
$$

because the coefficient $\left\langle{ }_{p^{m}}^{2 p^{m}}\right\rangle$ is invertible by Lemma 2.3 below. Next, for $I=p^{m} \mathbf{1}_{i}+$ $p^{m} \mathbf{1}_{j}$ with $i \neq j$, it gives

$$
\bar{\tau}_{i}^{p^{m}} \otimes \bar{\tau}_{j}^{p^{m}}+\bar{\tau}_{j}^{p^{m}} \otimes \bar{\tau}_{i}^{p^{m}}=0 .
$$

For the other I's, the given relations are trivial, which shows (i) and (ii). Now, (iii) is a direct consequence of Proposition 1.18.

LEMMA 2.3.- If $i \geq p^{m}$, the number $\left\langle\begin{array}{c}i \\ p^{m}\end{array}\right\rangle$ is congruent to 1 modulo $p$.

Proof. Put $i=p^{m} q+r$ with $q$ a natural number and $0 \leq r<p^{m}$. Then, we have $\left\langle\begin{array}{c}i \\ p^{m}\end{array}\right\rangle=(1 / q)\left(\begin{array}{c}i \\ p^{m}\end{array}\right)$, and this equals

$$
\frac{1}{q} \frac{p^{m} q+r}{r} \frac{p^{m} q+r-1}{r-1} \ldots \frac{p^{m} q+1}{1} \frac{p^{m} q}{p^{m}} \frac{p^{m} q-1}{p^{m}-1} \ldots \frac{p^{m} q-p^{m}+r+1}{r+1} .
$$

For $0<k<p^{m}$, the number $\left(p^{m} q+k\right) / k$ is congruent to 1 modulo $p$. This shows the lemma.

\subsection{Formal Higher Poincaré lemma.}

Now, we establish the Poincaré lemma for the higher de Rham complex in the local situation specified in the top of this section. 
LEMMA 2.4.- The linearized higher de Rham complex $L \breve{\Omega}_{X / S}^{(m) \cdot \bullet}$ is a resolution of the direct sum of $p^{m n}$ copies of $\mathscr{O}_{X}$. More strongly, if $h: T \rightarrow X$ is a morphism of $S$-schemes, the $\mathscr{O}_{T}$-linear map

$$
\iota^{\prime}: \bigoplus_{I \in \mathfrak{B}_{n}^{(m)}} \mathscr{O}_{T} e_{I} \rightarrow h^{*}\left(L \breve{\Omega}_{X / S}^{(m), \bullet}\right) ; e_{I} \mapsto h^{*}\left(\tau^{\{l\}}\right)
$$

is a quasi-isomorphism, where $\mathfrak{B}_{n}^{(m)}$ denotes the set of elements $I=\left(i_{1}, \ldots, i_{n}\right) \in \mathbb{N}^{n}$ such that $i_{j} \leq p^{m}$ for all $j=1, \ldots, n$.

Proof. When $n=1$ we have to show that the sequence

$$
0 \rightarrow \bigoplus_{i=0}^{p^{m}-1} \mathscr{O}_{T} e_{i} \rightarrow \mathscr{O}_{T}\{\tau\}_{(m)} \rightarrow \mathscr{O}_{T}\{\tau\}_{(m)} \bar{\tau}^{p^{m}} \rightarrow 0
$$

is exact, where $\tau$ here denotes $h^{*}\left(\tau_{1}\right)$; the second morphism sends $\tau^{\{i\}}$ to zero if $i<$ $p^{m}$ and to $\left\langle\begin{array}{c}i \\ p^{m}\end{array}\right\rangle \bar{\tau}^{p^{m}}$ if $i \geq p^{m}$. Since $\left\langle\begin{array}{c}i \\ p^{m}\end{array}\right\rangle$ is invertible by Lemma 2.3, the exactitude follows.

For an arbitrary $n$, the morphism $\iota^{\prime}$ is the tensor product of that for the $(n-1)$ dimensional case and that for the 1-dimensional case. Since each term of these complexes is free, the proof is obtained by induction on $n$.

The following proposition is a direct consequence of Lemma 2.4.

PROPOSITION 2.5.- Using the isomorphism

$$
\beta: \bigoplus_{I \in \mathfrak{B}_{n}^{(m)}} \mathscr{O}_{X} e_{I} \rightarrow \bigoplus_{I \in \mathfrak{B}_{n}^{(m)}} \mathscr{O}_{X} e_{I} ; \quad e_{I} \mapsto \sum_{0 \leq J \leq I}\left(\begin{array}{l}
I \\
J
\end{array}\right) t^{J} e_{I-J},
$$

we define the morphism

$$
\iota:=\iota^{\prime} \circ \beta^{-1}: \bigoplus_{I \in \mathfrak{B}_{n}^{(m)}} \mathscr{O}_{X} e_{I} \rightarrow L \breve{\Omega}_{X / S}^{(m), \bullet}
$$

Then, this is a quasi-isomorphism.

\subsection{Higher Poincaré lemma.}

In this subsection, we "lift" the results in the previous subsection to the $m$-crystalline site.

Set

$$
\mathscr{F}:=\bigoplus_{I \in \mathfrak{B}_{n}^{(m)}} \mathscr{O}_{X} e_{I}
$$

and equip it the hyper $m$-PD stratification

$$
\mathscr{P}_{X / S}^{(m)} \otimes_{\mathscr{O}_{X}} \mathscr{F} \rightarrow \mathscr{F} \otimes_{\mathscr{O}_{X}} \mathscr{P}_{X / S}^{(m)} ; 1 \otimes e_{I} \mapsto e_{I} \otimes 1 .
$$

Let $F$ denote the $m$-crystal corresponding, by Proposition 1.13, to the module $\mathscr{F}$ and this hyper $m$-PD stratification. $F$ is obviously isomorphic to the direct sum of $p^{m n}$ copies of the structure sheaf $\mathscr{O}_{X / S}^{(m)}$. 
LEMMA 2.6.- The $\mathscr{O}_{X}$-linear morphism $\iota: \mathscr{F} \rightarrow L \breve{\Omega}_{X / S}^{(m), 0}=\mathscr{P}_{X / S}^{(m)}$ defined in Proposition 2.5 is compatible with the hyper m-PD stratifications on both sides.

Proof. This lemma states that the diagram

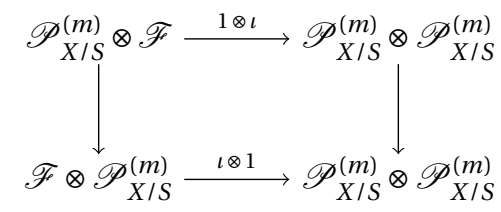

is commutative, where the right vertical morphism is calculated by using Proposition 1.19. In order to prove the commutativity, let us consider the section

$$
1 \otimes\left(\sum_{0 \leq J \leq I}\left(\begin{array}{l}
I \\
J
\end{array}\right) t^{J} e_{I-J}\right)
$$

of $\mathscr{P}_{X / S}^{(m)} \otimes \mathscr{F}$ for each $I \in \mathfrak{B}_{n}^{(m)}$; when $I$ runs through all elements in $\mathfrak{B}_{n}^{(m)}$, the sections (2.3.1) form a $\mathscr{P}_{X / S}^{(m)}$-basis of this module. This section (2.3.1) is sent by $1 \otimes \iota$ to $1 \otimes \tau^{I}$, and its image in the bottom is

$$
\sum_{0 \leq J \leq I}\left(\begin{array}{l}
I \\
J
\end{array}\right) \tau^{I-J} \otimes(-\tau)^{J}
$$

which is the image by $\iota \otimes 1$ of

$$
\sum_{0 \leq J \leq I}\left(\begin{array}{l}
I \\
J
\end{array}\right)\left\{\sum_{0 \leq K \leq I-J}\left(\begin{array}{c}
I-J \\
K
\end{array}\right) t^{K} e_{I-J-K}\right\} \otimes(-\tau)^{J}
$$

(note that $\left\langle\begin{array}{l}I \\ V\end{array}\right\rangle=\left(\begin{array}{l}I \\ V\end{array}\right)$ and that $\tau^{\{V\}}=\tau^{V}$ etc. because $I \in \mathfrak{B}_{n}^{(m)}$ ). On the other hand, (2.3.1) goes down to the section

$$
\sum_{0 \leq J \leq I}\left(\begin{array}{l}
I \\
J
\end{array}\right) e_{I-J} \otimes t^{J}
$$

Therefore, the problem is showing that the two sections (2.3.2) and (2.3.3) are identical. Now, the section (2.3.2) equals

$$
\sum_{0 \leq L \leq I}\left(\begin{array}{l}
I \\
L
\end{array}\right) e_{I-L}\left\{\sum_{0 \leq J \leq L}\left(\begin{array}{l}
L \\
J
\end{array}\right)(t \otimes 1)^{L-J}(-\tau)^{J}\right\}
$$

by changing the variables as $L=J+K$. The section of $\mathscr{P}_{X / S}^{(m)}$ in the braces is the canonical image of the section

$$
\sum_{0 \leq J \leq L}\left(\begin{array}{l}
L \\
J
\end{array}\right)(t \otimes 1)^{L-J}(1 \otimes t-t \otimes 1)^{J}=(1 \otimes t)^{L}
$$

of $\mathscr{O}_{X_{/ S}^{(2)}}$. This completes the proof.

Now, we are ready to prove the higher Poincaré lemma. 
THEOREM 2.7.- Let $M$ be an $\mathscr{O}_{X / S}^{(m)}$-module. Then, $M \otimes_{\mathscr{O}_{X / S}^{(m)}} L^{(m)}\left(\breve{\Omega}_{X / S}^{(m), \bullet}\right)$ forms a complex of $\mathscr{O}_{X / S}^{(m)}$-modules that resolves the direct sum of $p^{m n}$ copies of $M$.

Proof. First, we have a complex $L^{(m)}\left(\breve{\Omega}_{X / S}^{(m), \bullet}\right)$ of $\mathscr{O}_{X / S}^{(m)}$-modules that gives $L \breve{\Omega}_{X / S}^{(m), \bullet}$ on $X$; each term is, in fact, calculated by Proposition 1.15 (i), and each differential of $L \breve{\Omega}_{X / S}^{(m), \bullet}$ is compatible with the hyper $m$-PD stratification on each term by Lemma 1.20. Moreover, Lemma 2.6 ensures the $\mathscr{O}_{X / S}^{(m)}$-linear map $F \rightarrow L^{(m)}\left(\breve{\Omega}_{X / S}^{(m), 0}\right)$, whose composition with the differential map $L^{(m)}\left(\breve{\Omega}_{X / S}^{(m), 0}\right) \rightarrow L^{(m)}\left(\breve{\Omega}_{X / S}^{(m), 1}\right)$ is zero by Proposition 2.5. By tensoring $M$ over $\mathscr{O}_{X / S}^{(m)}$, we get a morphism

$$
M \otimes F \rightarrow M \otimes L^{(m)}\left(\breve{\Omega}_{X / S}^{(m), \bullet}\right) .
$$

We show that this is a quasi-isomorphism.

It suffices to argue on each $m$-PD thickening $(U, T, J, \delta)$ in $\operatorname{Cris}^{(m)}(X / S)$, and then the assertion is local on $T$. We may therefore assume that there exists an $S$ morphism $h: T \rightarrow X$ compatible with $i: U \hookrightarrow T$. Then on $T$, the map (2.3.4) is written as

$$
\bigoplus_{I \in \mathfrak{B}_{n}^{(m)}} M_{T} e_{I} \rightarrow M_{T} \otimes_{\mathscr{O}_{T}} h^{*}\left(L \check{\Omega}_{X / S}^{(m), \bullet}\right) .
$$

Now, Proposition 2.5 shows that

$$
\bigoplus_{I \in \mathfrak{B}_{n}^{(m)}} \mathscr{O}_{T} e_{I}=\mathscr{O}_{T} \otimes_{\mathscr{O}_{X}} \mathscr{F} \rightarrow h^{*}\left(L \breve{\Omega}_{X / S}^{(m), \bullet}\right)
$$

is quasi-isomorphic, and even after $M_{T}$ is tensored, it remains quasi-isomorphic because each term is locally free. This shows the assertion.

COROLlary 2.8.- Let $(S, \mathfrak{a}, \mathfrak{b}, \gamma)$ be an $m$-PD scheme and $X$ a smooth $S$-scheme that has global coordinates ${ }^{(* a)}$. Let $E$ be an $m$-crystal in $\mathscr{O}_{X / S}^{(m)}$-modules. Then, there exists an isomorphism in the derived category

$$
\left(\mathbb{R} u_{X / S *}^{(m)}(E)\right)^{\oplus p^{m n}} \rightarrow E_{X} \otimes_{\mathscr{O}_{X}} \breve{\Omega}_{X / S}^{(m), \bullet}
$$

Proof. The previous theorem, after the functor $\mathbb{R} u_{X / S *}^{(m)}$ is applied, shows that it suffices to prove that

$$
\mathbb{R} u_{X / S *}^{(m)}\left(E \otimes L^{(m)}\left(\breve{\Omega}_{X / S}^{(m), \bullet}\right)\right)=E_{X} \otimes \breve{\Omega}_{X / S}^{(m), \bullet},
$$

and we know from Proposition 1.15 (ii) and (iii) that this is true as graded $\mathscr{P}_{X / S^{-}}^{(m)}$ modules. Since this identification is via the map

$$
d^{0^{*}}\left(E_{X} \otimes \breve{\Omega}_{X / S}^{(m), \bullet}\right) \rightarrow \mathscr{P}_{X / S}^{(m)} \otimes E_{X} \otimes \breve{\Omega}_{X / S}^{(m), \bullet},
$$

we see that the differential maps on both sides coincide because of the relations of $d_{i}^{*}$ 's.

${ }^{(* a)}$ This assumption, which we have always kept in this section, is written here just for the convienience of the reader. 


\section{Finiteness of Cohomology.}

\subsection{Cohomological Boundedness.}

Now, we are going to use the higher Poincaré lemma to prove the boundedness, the base change and the finiteness of the crystalline cohomology of level $\mathrm{m}$.

THEOREM 3.1.- Let $(S, \mathfrak{a}, \mathfrak{b}, \gamma)$ be an m-PD scheme, $\left(\mathfrak{a}_{0}, \mathfrak{b}_{0}, \gamma_{0}\right)$ be a quasi-coherent $m$-PD sub-ideal of $\mathfrak{a}$. Let $X$ be a smooth scheme over $S_{0}$, and assume that the structure morphism $f: X \rightarrow S$ is quasi-compact and quasi-separated; moreover, we assume that $S$ is quasi-separated. Then, for each quasi-coherent $\mathscr{O}_{X / S}^{(m)}$-module E and each natural number $i$, the $\mathscr{O}_{S}$-module $R^{i} f_{X / S_{*}}^{(m)} E$ is quasi-coherent. Moreover, there exists an integer $r$ such that $R^{i} f_{X / S *}^{(m)} E=0$ for all $i>r$ and for all quasi-coherent $\mathscr{O}_{X / S^{-}}^{(m)}$ module E.

Proof. First, if $X$ can be lifted to a smooth $S$-scheme having local coordinates, then Corollary 2.8 and Proposition 1.11 show the assertions.

Next, we assume that $X$ is separated. Let $U=\left(U_{i}\right)$ be a finite covering by affine open subschemes of $X$ which have local coordinates. For each natural number $v$, put

$$
U_{(v)}:=\coprod_{i_{0}<\cdots<i_{v}} U_{i_{0}} \cap \cdots \cap U_{i_{v}}
$$

and let $j_{(v)}$ denote the natural map $U_{(v)} \rightarrow X$. Then [B-O, 7.6], there exists a spectral sequence

$$
E_{1}^{p, q}=R^{q} f_{U_{(p)} / S_{*}}^{(m)} j_{(p)}^{*}(E) \Longrightarrow R^{n} f_{X / S *}^{(m)} E
$$

by which the assertions are reduced to those for each $U_{i_{0}} \cap \cdots \cap U_{i_{p}}$. These schemes in turn are affine and have local coordinates by assumption, therefore the proof is finished in this case.

At last, for general $X$, we repeat the same argument; the schemes $U_{i_{0}} \cap \cdots \cap U_{i_{p}}$ constructed as above are not necessarily affine, but are quasi-compact and quasiaffine, therefore separated.

\subsection{Base Change Theorem.}

THEOREM 3.2.- Let $u:\left(S^{\prime}, \mathfrak{a}^{\prime}, \mathfrak{b}^{\prime}, \gamma^{\prime}\right) \rightarrow(S, \mathfrak{a}, \mathfrak{b}, \gamma)$ be a morphism of $m$-PD schemes, $Y$ (resp. $Y^{\prime}$ ) a scheme over $S$ (resp. $S^{\prime}$ ) and $h: Y^{\prime} \rightarrow Y$ an $S$-morphism. We assume that $Y$ is quasi-compact. Let $f: X \rightarrow Y$ be a smooth morphism, and let $f^{\prime}: X^{\prime} \rightarrow Y^{\prime}$ denote the base change of $f$ over $h$, and let $g$ denote the projection $X^{\prime} \rightarrow X$. Then, if $E$ is a flat and quasi-coherent $\mathscr{O}_{X / S}^{(m)}$-module, there exists an isomorphism

$$
\mathbb{L} h_{\text {cris }}^{(m) *} R f_{\text {cris }}^{(m)}(E) \rightarrow R f_{\text {cris }}^{\prime(m)} g_{\text {cris }}^{(m) *}(E) .
$$




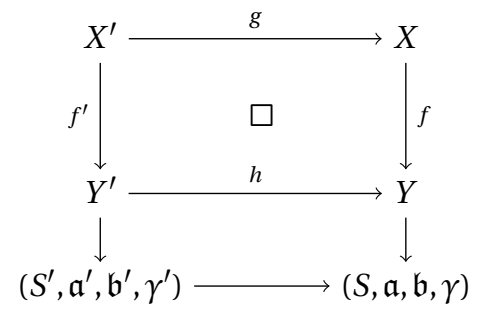

Proof. Because $\mathbb{R} f_{\text {cris* }}^{(m)}(E)$ is bounded by Theorem 3.1, the complex in the left hand side makes sense. Then, we may construct the base change morphism using the adjunction formula [B1, V 3.3.1]. In order to prove that this morphism is isomorphic, following the argument for classical case [B1, V 3.5.5], we know that it suffices to prove the following weaker proposition.

Proposition 3.3.- In the situation in Theorem 3.2, let $\left(\mathfrak{a}_{0}, \mathfrak{b}_{0}, \gamma_{0}\right)\left(\operatorname{resp} .\left(\mathfrak{a}_{0}^{\prime}, \mathfrak{b}_{0}^{\prime}, \gamma_{0}^{\prime}\right)\right)$ be a quasi-coherent $m$-PD sub-ideal of $\mathfrak{a}\left(\right.$ resp. $\left.\mathfrak{a}^{\prime}\right)$, and assume that $Y$ (resp. $Y^{\prime}$ ) is the closed subscheme $S_{0}$ (resp. $S_{0}^{\prime}$ ) of $S$ (resp. $S^{\prime}$ ) defined by the ideal $\mathfrak{a}_{0}$ (resp. $\mathfrak{a}_{0}^{\prime}$ ). We rename the morphisms as in the diagram below. Then, if $E$ is a flat and quasi-coherent $\mathscr{O}_{X / S}^{(m)}$-module, the base changing map

$$
\llbracket u^{*} R f_{X / S *}^{(m)}(E) \rightarrow R f_{X^{\prime} / S^{\prime} *}^{(m)} g_{\text {cris }}^{(m) *}(E)
$$

is an isomorphism.

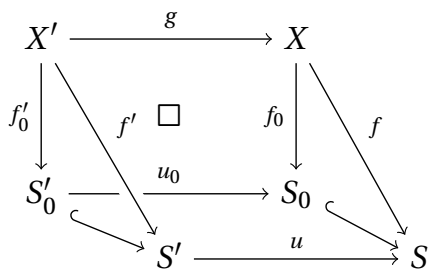

Proof. At first, we assume that $X$ is lifted to a scheme $Y$ which is affine, is smooth of relative dimension $n$ over $S$, and has local coordinates. Then by Corollary 1.14, we may assume that $X=Y$.

Now, by Corollary 2.8, we have an isomorphism

$$
\left(\mathbb{R} f_{X / S *}^{(m)}(E)\right)^{\oplus p^{m n}} \stackrel{\sim}{\longrightarrow} f_{*}\left(E_{X} \otimes \breve{\Omega}_{X / S}^{(m), \bullet}\right)
$$

and $E^{\prime}:=g_{\text {cris }}^{(m) *}(E)$ in turn satisfies

$$
\left(\mathbb{R} f_{X^{\prime} / S^{\prime} *}^{\prime(m)}\left(E^{\prime}\right)\right)^{\oplus p^{m n}} \stackrel{\longrightarrow}{\longrightarrow} f_{*}^{\prime}\left(E_{X^{\prime}}^{\prime} \otimes \check{\Omega}_{X^{\prime} / S^{\prime}}^{(m), \bullet} .\right.
$$

In the right-hand side of (3.2.2) (resp. (3.2.3)), the functor $\mathbb{R} f_{*}\left(\right.$ resp. $\mathbb{R} f_{*}^{\prime}$ ) can be replaced by $f_{*}$ (resp. $f_{*}^{\prime}$ ) because of the quasi-coherence of $E$ (resp. $E^{\prime}$ ). We then 
have a commutative diagram

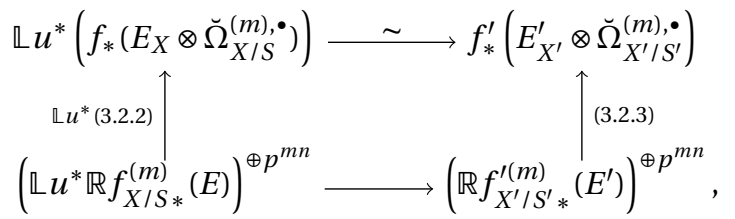

where the lower horizontal morphism is the direct sum of $p^{m n}$ copies of (3.2.1). The upper horizontal morphism exists because the local coordinates of $X$ over $S$ and those of $X^{\prime}$ over $S^{\prime}$ are compatible, and it is clear that this is an isomorphism.

This completes the proof for this special case. In the general case, we can use the descent argument [B1, pp.344-347].

\subsection{Finiteness.}

THEOREM 3.4.- Let $(S, \mathfrak{a}, \mathfrak{b}, \gamma)$ be an m-PD scheme and $\left(\mathfrak{a}_{0}, \mathfrak{b}_{0}, \gamma_{0}\right)$ a quasi-coherent $m-P D$ sub-ideal of $\mathfrak{a}$. The closed subscheme of $S$ defined by $\mathfrak{a}_{0}$ is denoted by $S_{0}$. Assume that $X$ is a smooth proper scheme over $S_{0}$ and that $S$ is noetherian. Let $f$ denote the structure morphism of $X$ over $S$. In this situation, if $E$ is a locally free $\mathscr{O}_{X / S}^{(m)}$-module of finite rank, then $\mathbb{R} f_{X / S *}^{(m)} E$ is a perfect complex of $\mathscr{O}_{S}$-modules.

Proof. The assertion is equivalent to showing that the complex of $\mathscr{O}_{S}$-modules $\mathbb{R} f_{X / S *}^{(m)} E$ is pseudo-coherent and locally of finite Tor-dimension [SGA6, I 5.8.1] (recall that a complex $K$ of $\mathscr{O}_{S}$-modules is pseudo-coherent if and only if it is locally of finite cohomological dimension and the cohomology sheaves $H^{i}(K)$ are coherent for all $i$ [SGA6, I p.2]). In fact, we can deduce from Proposition 3.3 that $\mathbb{R} f_{X / S_{*}}^{(m)} E$ is locally of finite Tor-dimension [B1, V 3.5.9]. It therefore suffices to prove the pseudocoherence of this complex.

First, in case $\mathfrak{a}_{0}=0$, that is, in case $S=S_{0}$, this complex is quasi-isomorphic to a complex whose terms are finitely generated [LS-Q2, 4.7] (as explained in Subsection 0.2 , no problem occurs in the use of this result of Le Stum and Quirós because of the local freeness of $E$ ). Hence the pseudo-coherence is obvious.

Next, assume that $\left(\mathfrak{a}_{0}, \mathfrak{b}_{0}, \gamma_{0}\right)=(\mathfrak{a}, \mathfrak{b}, \gamma)$. In this case, the argument [B1, VII 1.1.1] is used as follows. For each natural number $n$, let $S_{n}$ be the closed subscheme defined by $\mathfrak{a}^{n+1}$. Then, we have the exact sequence

$$
0 \rightarrow \mathfrak{a}^{n} / \mathfrak{a}^{n+1} \rightarrow \mathscr{O}_{S_{n}} \rightarrow \mathscr{O}_{S_{n-1}} \rightarrow 0,
$$

which gives the distinguished triangle

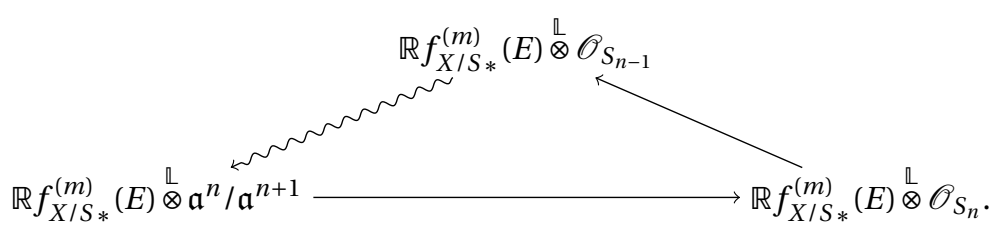


By Proposition 3.3, the complex in the left-bottom is isomorphic to

$$
\mathbb{R} f_{X / S_{0 *}}^{(m)}(E)^{\mathbb{R}} \mathscr{O}_{S_{0}} \mathfrak{a}^{n} / \mathfrak{a}^{n+1},
$$

which is pseudo-coherent by the case where $\mathfrak{a}_{0}=0$. Therefore [SGA6, I $2.5 \mathrm{~b}$ )], noting that the ideal $\mathfrak{a}_{0}$ is nilpotent, the proof is obtained by induction.

At last, we consider the general case. Then, the category of the $m$-crystals in $\mathscr{O}_{X /(S, \mathfrak{a}, \mathfrak{b}, \gamma)}^{(m)}$-modules and that of the $m$-crystals in $\mathscr{O}_{X /\left(S, \mathfrak{a}_{0}, \mathfrak{b}_{0}, \gamma_{0}\right)}^{(m)}$-modules are equivalent; indeed, the question being local on $X$, we may assume that $X$ is embedded to a smooth scheme $Y$ over $S$ such that $Y \times{ }_{S} S_{0}=X$, and then Proposition 1.13 applies. Thus the theorem is reduced to the previous case by using Proposition 3.3.

\section{Other Applications.}

\subsection{Künneth formula.}

THEOREM 4.1.- Let $(S, \mathfrak{a}, \mathfrak{b}, \gamma)$ be an $m$-PD scheme, and $R$ a quasi-compact $S$ scheme. Let $f: X \rightarrow R$ and $g: Y \rightarrow R$ be two quasi-compact, quasi-separated and smooth morphisms. Denote the fiber product $X \times_{R} Y$ by $Z$, the projection $Z \rightarrow X$ (resp. $Z \rightarrow Y$ ) by $p$ (resp. by $q$ ) and the morphism $f \circ p=g \circ q$ by $h$;

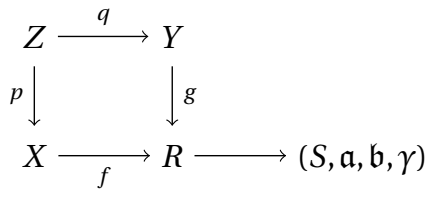

If $E$ (resp. $F$ ) is a quasi-coherent and flat $\mathscr{O}_{X / S}^{(m)}$-module (resp. $\mathscr{O}_{Y / S}^{(m)}$-module), then there exists an isomorphism

$$
\mathbb{R} f_{\text {cris } *}^{(m)}(E) \stackrel{\mathbb{Q}}{\otimes}_{\mathscr{O}_{R / S}^{(m)}} \mathbb{R} g_{\text {cris } *}^{(m)}(F) \longrightarrow \mathbb{R} h_{\text {cris } *}^{(m)}\left(p_{\text {cris }}^{(m)^{*}}(E) \otimes_{\mathscr{O}_{Z / S}^{(m)}} q_{\text {cris }}^{(m)^{*}}(F)\right)
$$

Proof. The construction of this morphism is straightforward by using the adjunction formula [B1, V 4.1.1]. In order to prove that the morphism is an isomorphim, the following proposition will suffice.

Proposition 4.2.- Under the situation in Theorem 4.1, let $\left(\mathfrak{a}_{0}, \mathfrak{b}_{0}, \gamma_{0}\right)$ be a quasicoherent $m-P D$ sub-ideal of $\mathfrak{a}$, and assume that $R$ is the closed subscheme $S_{0}$ of $S$ defined by $\mathfrak{a}_{0}$. Then, the morphism

$$
\mathbb{R} f_{X / S *}^{(m)}(E) \stackrel{\mathbb{R}}{\otimes} \mathscr{O}_{S} \mathbb{R} g_{X / S *}^{(m)}(F) \longrightarrow \mathbb{R} h_{Z / S *}^{(m)}\left(p_{\text {cris }}^{(m)^{*}}(E) \otimes_{\mathscr{O}_{Z / S}^{(m)}} q_{\text {cris }}^{(m)^{*}}(F)\right)
$$

is an isomorphism.

Proof. We may assume that $X$ (resp. $Y$ ) is lifted to an affine smooth $S$-scheme $\bar{X}$ (resp. $\bar{Y}$ ) that has local coordinates. Then, $\bar{X} \times{ }_{S} \bar{Y}$ is a lift of $Z$, which allows us to assume that $\bar{X}=X, \bar{Y}=Y$ and $Z=\bar{X} \times{ }_{S} \bar{Y}$. We fix a system of local coordinates 
$\left\{t_{1}, \ldots, t_{n}\right\}$ (resp. $\left\{t_{1}^{\prime}, \ldots, t_{n^{\prime}}^{\prime}\right\}$ ) of $X$ (resp. $Y$ ); the scheme $Z$ naturally has a system of local coordinates

$$
\left\{p^{*}\left(t_{1}\right), \ldots, p^{*}\left(t_{n}\right), q^{*}\left(t_{1}^{\prime}\right), \ldots, q^{*}\left(t_{n^{\prime}}^{\prime}\right)\right\},
$$

which gives us a natural isomorphism

$$
p^{*}\left(\breve{\Omega}_{X / S}^{(m), \bullet}\right) \otimes q^{*}\left(\breve{\Omega}_{Y / S}^{(m), \bullet}\right) \stackrel{\sim}{\longrightarrow} \breve{\Omega}_{Z / S}^{(m), \bullet}
$$

The proposition, therefore, follows from the commutative diagram

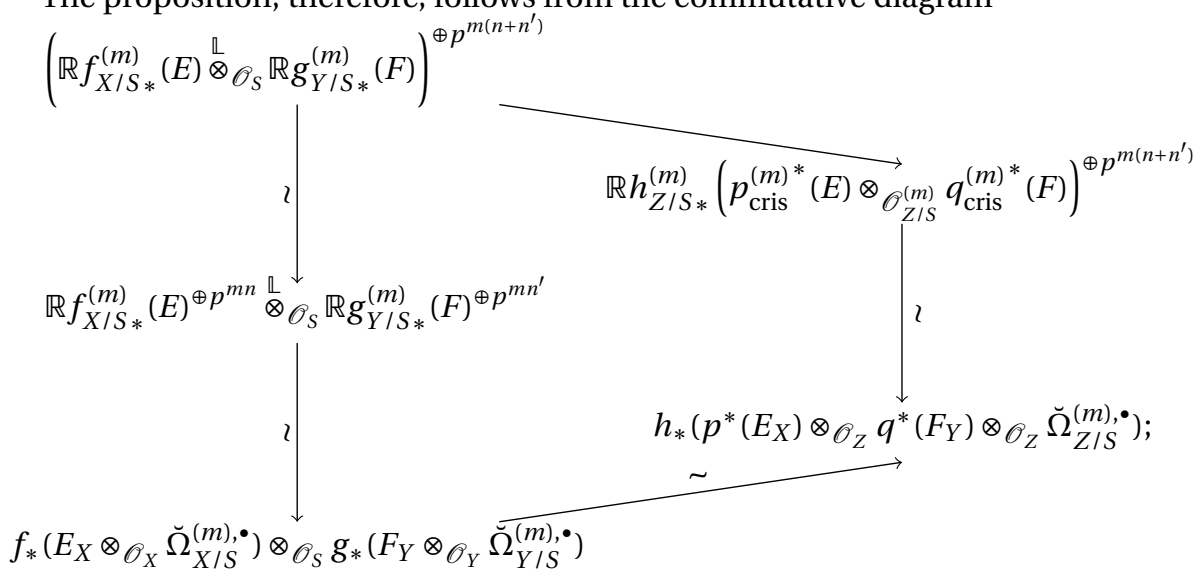

here, the morphism from the top-left toward bottom-right is the direct sum of $p^{m\left(n+n^{\prime}\right)}$ copies of (4.1.1), and that from the top-left toward bottom is induced from

$$
\begin{aligned}
\left(f_{\text {cris } *}^{(m)}(E) \otimes g_{\text {cris } *}^{(m)}(F)\right)^{\oplus p^{m\left(n+n^{\prime}\right)}} & \rightarrow f_{\text {cris } *}^{(m)}(E)^{\oplus p^{m n}} \otimes g_{\text {cris } *}^{(m)}(F)^{\oplus p^{m n^{\prime}}} \\
e_{Z,\left(I, I^{\prime}\right)} & \mapsto e_{X, I} \otimes e_{Y, I^{\prime}} .
\end{aligned}
$$

where $I \in \mathfrak{B}_{n}^{(m)}, I^{\prime} \in \mathfrak{B}_{n^{\prime}}^{(m)}$ and $e_{X, I}$ (resp. $\left.e_{Y, I^{\prime}}, e_{Z,\left(I, I^{\prime}\right)}\right)$ is the canonical basis of each direct sum.

\subsection{Frobenius Descent.}

In this subsection, we correct the proof [LS-Q2, 5] of Frobenius descent.

LEMMA 4.3.- Let $(S, \mathfrak{a}, \mathfrak{b}, \gamma)$ be an $m$-PD scheme, let $X$ be an $n$-dimensional smooth scheme over $S$ that has local coordinates, and let $\mathscr{F}$ be a $\mathscr{D}_{X / S}^{(m)}$-module. Then, there exists a natural isomorphism

$$
\mathscr{F} \otimes_{\mathscr{O}_{X}} \breve{\Omega}_{X / S}^{(m), \bullet} \stackrel{\sim}{\longrightarrow} \mathscr{H} O m_{\mathscr{D}_{X / S}^{(m)}}\left(\mathscr{O}_{X}, \mathscr{F}\right)^{\oplus p^{m n}}
$$

Proof. Because each term of the higher de Rham complex is free, the complex

$$
\mathscr{H}_{0 m_{O_{X}}}\left(\breve{\Omega}_{X / S}^{(m), \bullet}, \mathscr{D}_{X / S}^{(m)}\right)
$$


gives a free resolution of $\mathscr{O}_{X}^{\oplus p^{m n}}$ as $\mathscr{D}_{X / S}^{(m)}$-modules. Because of the freeness again, the morphism

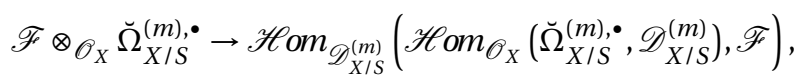

is an isomorphism, which completes the proof.

In the remainder of this subsection, let $(S, \mathfrak{a}, \mathfrak{b}, \gamma)$ be an $m$-PD scheme, let $\left(\mathfrak{a}_{0}, \mathfrak{b}_{0}, \gamma_{0}\right)$ a quasi-coherent $m$-PD sub-ideal of $\mathfrak{a}$ that satisfies $p \in \mathfrak{a}_{0}$, and denote by $S_{0}$ the closed subscheme defined by $\mathfrak{a}_{0}$. Let $f: X_{0} \rightarrow S_{0}$ be a smooth morphism such that the $m$-PD structure $\left(\mathfrak{b}_{0}, \gamma_{0}\right)$ extends to $\mathscr{O}_{X_{0}}$, and let $F_{0}: X_{0} \rightarrow X_{0}^{\prime}$ denote the $s$-th iterate of the relative Frobenius of $f$ for a natural number $s$. We denote by $f^{\prime}: X_{0}^{\prime} \rightarrow S_{0}$ the natural morphism.

Under this situation, the morphism $F_{0}$ induces a morphism of topoi [B2, (4.1)]

$$
F_{0}:\left(X_{0} / S\right)_{\text {cris }}^{(m+s)} \rightarrow\left(X_{0}^{\prime} / S\right)_{\text {cris }}^{(m)} .
$$

In order to recall the construction of the inverse image functor of $F_{0}$, we fix an object $(U, T, J, \delta)$ of $\mathrm{Cris}^{(m+s)}\left(X_{0} / S\right)$ and set some notation. Let $T_{0}$ denote the closed subscheme of $T$ defined by $J+p \mathscr{O}_{T}$, and $T_{1}$ the closed subscheme of $T_{0}$ defined by $I^{\left(p^{m}\right)} \mathscr{O}_{T_{0}}$, where $I$ denotes the ideal of $\mathscr{O}_{T}$ defined by the closed immersion $U \hookrightarrow T$. We denote the $s$-th iterate of the relative Frobenius morphism of $U$ (resp. $T_{1}, T_{0}$ ) by $F_{0, U}: U \rightarrow U^{\prime}$ (resp. $F_{0, T_{1}}: T_{1} \rightarrow T_{1}^{\prime}, F_{0, T_{0}}: T_{0} \rightarrow T_{0}^{\prime}$ ). Now, we have a continuous functor

$$
\operatorname{Cris}^{(0)}\left(T_{1}^{\prime} / S\right) \rightarrow \operatorname{Cris}^{(m)}\left(X^{\prime} / S\right)
$$

defined as follows. For an object $\left(W, V, \delta_{0}\right)$ in $\operatorname{Cris}^{(0)}\left(T_{1}^{\prime} / S\right)$, if $I_{0}$ denotes the ideal of $\mathscr{O}_{V}$ defined by the closed immersion $W \hookrightarrow V$, the image of $\left(W, V, \delta_{0}\right)$ through the functor above is $\left(U^{\prime} \cap W, V, I_{0}, \delta_{0}\right)$; in fact, $\left(I_{0}, \delta_{0}\right)$ is the $m$-PD structure of the ideal of the closed immersion $U^{\prime} \cap W \hookrightarrow W \hookrightarrow V$ since, because $\left(I^{\left(p^{m}\right)} \mathscr{O}_{T_{0}}\right)^{\left(p^{s}\right)}=0$, we have $T_{0}^{\prime}=T_{1}^{\prime}$. We may directly prove that this defines a continuous functor.

Now, for a sheaf $E$ on $\mathrm{Cris}^{(m)}\left(X_{0}^{\prime} / S\right)$, the section of $F_{0}^{*} E$ on $(U, T, J, \delta)$ is described as follows. If $E_{1}$ denotes the sheaf on $\mathrm{Cris}^{(0)}\left(T_{1}^{\prime} / S\right)$ induced by $E$, and if $E_{2}$ denotes the image of $E_{1}$ through the inverse image functor of $\left(F_{0, T_{0}}\right)_{\mathrm{cris}}^{(0)}$, we have

$$
\left(F_{0}^{*} E\right)(U, T, J, \delta)=E_{2}\left(T_{0}, T\right) .
$$

If $E$ is an $m$-crystal in $\mathscr{O}_{X_{0}^{\prime} / S}^{(m)}$-modules, then $F_{0}^{*} E$ is an $(m+s)$-crystal in $\mathscr{O}_{X^{\prime} / S}^{(m+s)}$ modules; in fact, the question being local, we may assume that $X_{0}$ (resp. $X_{0}^{\prime}$ ) can be lifted into a smooth $S$-scheme $X$ (resp. $X^{\prime}$ ) and $F_{0}$ can be lifted into a morphism $F: X \rightarrow X^{\prime}$, in which case $F_{0}^{*} E$ is an $(m+s)$-crystal [B4, 2.2.3].

Proposition 4.4.- Let $E^{\prime}$ be a locally free $\mathscr{O}_{X_{0}^{\prime} / S}^{(m)}$-module of finite rank, and denote by $E$ the $\mathscr{O}_{X_{0} / S}^{(m+s)}$-module $F_{0}^{*} E^{\prime}$, which is an $(m+s)$-crystal as noted above.

Then, the morphism

$$
F_{0}^{*}: R u_{X_{0}^{\prime} / S_{*}}^{(m)}\left(E^{\prime}\right) \rightarrow R u_{X_{0} / S}^{(m+s)}(E)
$$

is an isomorphism. 
Before starting the proof, we show the following lemma.

LemMA 4.5.- Assume that $p \mathscr{O}_{S}=0$, that $\mathfrak{a}_{0}=0$ and let us omit the subscripts " 0 " in the notation. Moreover, assume that $X$ and $X^{\prime}$ has a system of local coordinates; we fix one $\left\{t_{1}, \ldots, t_{n}\right\}$ of $X$ and $\left\{t_{1}^{\prime}, \ldots, t_{n}^{\prime}\right\}$ of $X^{\prime}$.

Then, the morphism $F: X^{\prime} \rightarrow X$ induces a quasi-isomorphism

$$
\varphi: E_{X^{\prime}}^{\prime} \otimes\left(\breve{\Omega}_{X^{\prime} / S}^{(m), \bullet}\right)^{\oplus p^{s n}} \rightarrow E_{X} \otimes \check{\Omega}_{X / S}^{(m+s), \bullet} .
$$

Proof. Since Berthelot [B4, 2.2.2 (i)] shows that $F$ induces a morphism of simplicial schemes

$$
F^{*}: P_{X^{\prime} / S}^{(m)}(\bullet) \rightarrow P_{X / S}^{(m+s)}(\bullet)
$$

we get a morphism

$$
F^{*}: E_{X^{\prime}}^{\prime} \otimes \mathscr{P}_{X^{\prime} / S}^{(m), \bullet} \rightarrow E_{X} \otimes \mathscr{P}_{X / S}^{(m+s), \bullet} .
$$

We show that this induces a morphism

$$
F^{*}: E_{X^{\prime}}^{\prime} \otimes \breve{\Omega}_{X^{\prime} / S}^{(m), \bullet} \rightarrow E_{X} \otimes \breve{\Omega}_{X / S}^{(m+s), \bullet} .
$$

Put $\tau_{i}=d^{*}\left(t_{i}\right)$ and $\tau_{i}^{\prime}=d^{*}\left(t_{i}^{\prime}\right)$, and recall that [B4, 2.2.4 (i)], for each $I \in \mathbb{N}^{n} \backslash\{0\}$, this morphism takes $\tau^{\prime\{I\}}$ to $\tau^{\left\{p^{s} I\right\}}$. Therefore, (4.2.3) induces

$$
E_{X^{\prime}}^{\prime} \otimes N \mathscr{P}_{X^{\prime} / S}^{(m), \bullet} \rightarrow E_{X} \otimes N \mathscr{P}_{X / S}^{(m), \bullet} .
$$

Moreover, if $I$ is not equal to $p^{m} \mathbf{1}_{i}$ for any $i=1, \ldots, n$, then $p^{s} I$ is not of the form $p^{m+s} \mathbf{1}_{j}$, which shows that the image of $\mathscr{K}_{X^{\prime} / S}^{(m)}$ under $F^{*}$ lies in $\mathscr{K}_{X / S}^{(m+s)}$.

Now, for each $J \in \mathfrak{B}_{s}^{(m)}$, consider the morphism

$$
F_{J}: E_{X^{\prime}}^{\prime} \otimes \breve{\Omega}_{X^{\prime} / S}^{(m), \bullet} \rightarrow E_{X} \otimes \breve{\Omega}_{X / S}^{(m+s), \bullet}
$$

obtained by multiplying $\tau^{J}$ from the left after $F^{*}$ above. This morphism is actually a zero morphism if $J \neq 0$ since $\tau^{J} \tau_{i}^{p^{m+s}}=0$ in $\breve{\Omega}_{X / S}^{(m+s), 1}$. We define the morphism $\varphi$ to be the direct sum of $F_{J}$ 's for $J \in \mathfrak{B}_{s}^{(m)}$.

The morphism $\varphi$ fits into the commutative diagram

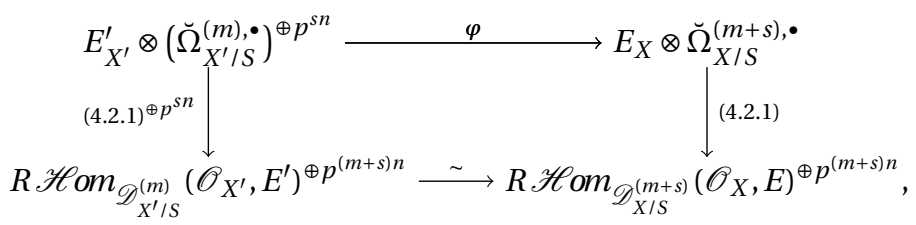

where the morphism in the bottom is the direct sum of $p^{(m+s) n}$ copies of

$$
F^{*}: R \mathscr{H}_{\mathscr{D}_{X^{\prime} / S}^{(m)}}\left(\mathscr{O}_{X^{\prime}}, E_{X^{\prime}}\right) \rightarrow R \mathscr{H} O m_{\mathscr{D}_{X / S}^{(m+s)}}\left(\mathscr{O}_{X}, E_{X}\right)
$$

this is a quasi-isomorphism by Berthelot [B4, 2.3 .8 (i)], which shows the lemma.

Proof of Proposition 4.4. First, we reduce the proof to the case where the assumptions in the previous lemma holds. The question being local, we may assume that $X_{0}\left(\right.$ resp. $X_{0}^{\prime}$ ) can be lifted into a smooth $S$-scheme $X$ (resp. $X^{\prime}$ ) and $F_{0}$ can be lifted into a morphism $F: X \rightarrow X^{\prime}$, in which case Corollary 1.14 and Proposition 3.3 allows us to assume that $\left(\mathfrak{a}_{0}, \mathfrak{b}_{0}, \gamma_{0}\right)=(\mathfrak{a}, \mathfrak{b}, \gamma)$. Then, by using the distinguished triangle as 
in the proof of Theorem 3.4, we may assume that $p \mathscr{O}_{S}=0$ and $\mathfrak{a}=0$. Finally, again since the question is local, we may argue under the situation of the previous lemma.

Now, the construction shows that the following diagram commutes:

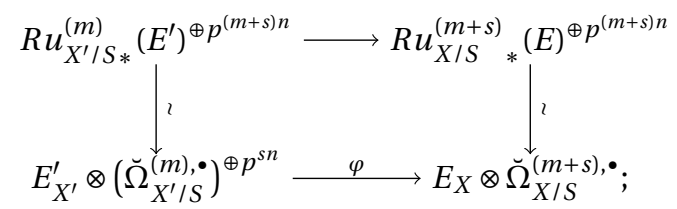

here, the morphism in the top is the direct sum of $p^{(m+s) n}$ copies of the morphism (4.2.2). This shows the proposition.

\section{References}

[SGA4] P. Berthelot, A. Grothendieck and L. Illusie, “Théorie des Topos et Cohomologie Étale des Schémas," Lecture Notes in Math. 269, 270, 305, Springer-Verlag.

[SGA6] M. Artin, A. Grothendieck and J.-L. Verdier, "Théorie des Intersections et théorème de Riemann-Roch," Lecture Notes in Math. 225, Springer-Verlag.

[B1] P. Berthelot, “Cohomologie Cristalline des Schémas de Caractéristique $p>0$," Lecture Notes in Math. 407, Springer-Verlag, 1974.

[B2] P. Berthelot, "Letter to illusie," 1990.

[B3] P. Berthelot, “ $\mathscr{D}_{\mathbb{Q}}^{\dagger}$-modules arithmétiques. I. Opérateurs différentiels de niveau fini,” Ann. Sci. Ecole Norm. Sup. 29 (1996), 185-272.

[B4] P. Berthelot, “DD-modules arithmétiques. II. Descente par Frobenius,” Mem. Soc. Math. France 81 (2000), 1-136.

[B5] P. Berthelot, Letter to Abe and the author, 2010.

[B-O] P. Berthelot and A. Ogus, "Notes on Crystalline Cohomology," Princeton University Press, 1978.

[LS-Q1] B. Le Stum and A. Quirós, “Transversal crystal of higher level," Ann. Inst. Fourier (Grenoble) 47(1) (1997), 69-100.

[LS-Q2] B. Le Stum and A. Quirós, “The exact Poincaré lemma in crystalline cohomology of higher level,” J. of Algebra 240 (2001), 559-588. 